\title{
Compositional synthesis of finite abstractions for networks of systems: A small-gain approach
}

\author{
Abdalla Swikir a,*, Majid Zamani b,c \\ a Department of Electrical and Computer Engineering, Technical University of Munich, Germany \\ ${ }^{\mathrm{b}}$ Department of Computer Science, University of Colorado Boulder, USA \\ c Department of Computer Science, Ludwig Maximilian University of Munich, Germany
}

\section{A R T I C L E I N F O}

\section{Article history:}

Received 16 May 2018

Received in revised form 15 February 2019

Accepted 13 June 2019

Available online 4 July 2019

\section{Keywords:}

Finite abstractions

Compositionality

Networks of systems

Symbolic control

Formal synthesis

Small-gain

\begin{abstract}
A B S T R A C T
In this paper, we introduce a compositional scheme for the construction of finite abstractions (a.k.a. symbolic models) of interconnected discrete-time control systems. The compositional scheme is based on small-gain type reasoning. In particular, we use a notion of so-called alternating simulation functions as a relation between each subsystem and its symbolic model. Assuming some smallgain type conditions, we construct compositionally an overall alternating simulation function as a relation between an interconnection of symbolic models and that of original control subsystems. In such compositionality reasoning, the gains associated with the alternating simulation functions of the subsystems satisfy a certain "small-gain" condition. In addition, we introduce a technique to construct symbolic models together with their corresponding alternating simulation functions for discrete-time control subsystems under some stability property. Finally, we apply our results to the temperature regulation in a circular building by constructing compositionally a finite abstraction of a network containing $N$ rooms for any $N \geq 3$. We use the constructed symbolic models as substitutes to synthesize controllers compositionally maintaining room temperatures in a comfort zone. We choose $N=1000$ for the sake of illustrating the results. We also apply our proposed techniques to a nonlinear example of a fully connected network in which the compositionality condition still holds for any number of components. In these case studies, we show the effectiveness of the proposed results in comparison with the existing compositionality technique in the literature using a dissipativity-type reasoning.
\end{abstract}

(C) 2019 Elsevier Ltd. All rights reserved.

\section{Introduction}

In general, designing complex systems with respect to sophisticated control objectives is a challenging problem. In the past few years, several techniques have been developed to overcome those challenges. One particular approach to address complex systems and control objectives is based on the construction of finite abstractions (a.k.a. symbolic models) of the original control systems. Finite abstractions provide abstract descriptions of the continuous-space control systems in which each discrete state

\footnotetext{
This work was supported in part by the H2020 ERC Starting Grant AutoCPS (grant agreement no 804639) and the TUM International Graduate School of Science and Engineering (IGSSE). The material in this paper was not presented at any conference. This paper was recommended for publication in revised form by Associate Editor Christoforos Hadjicostis under the direction of Editor Christos G. Cassandras.

* Corresponding author.

E-mail addresses: abdalla.swikir@tum.de (A. Swikir), majid.zamani@colorado.edu (M. Zamani).
}

and input correspond to an aggregate of continuous states and inputs of the original system, respectively.

In general, there exist two types of symbolic models: sound ones whose behaviors (approximately) contain those of the concrete systems and complete ones whose behaviors are (approximately) equivalent to those of the concrete systems (Tabuada, 2009). Remark that existence of a complete symbolic model results in a sufficient and necessary guarantee in the sense that there exists a controller enforcing the desired specifications on the symbolic model if and only if there exists a controller enforcing the same specifications on the original control system. On the other hand, a sound symbolic model provides only a sufficient guarantee in the sense that failing to find a controller for the desired specifications on the symbolic model does not prevent the existence of a controller for the original control system. Since symbolic models are finite, controller synthesis problems can be algorithmically solved over them by resorting to automatatheoretic approaches (Maler, Pnueli, \& Sifakis, 1995; Thomas, 1995). Unfortunately, the construction of symbolic models for large-scale interconnected systems is itself computationally a 
complex and challenging task. An appropriate technique to overcome this challenge is to first construct symbolic models of the concrete subsystems individually and then establish a compositional framework using which one can construct abstractions of the overall network using those individual abstractions.

In the past few years, there have been several results on the compositional construction of finite abstractions of networks of control subsystems. The framework introduced by Tazaki and Imura (2008) based on the notion of interconnection-compatible approximate bisimulation relation provides networks of finite abstractions approximating networks of stabilizable linear control systems. This work was extended by Pola, Pepe, and Benedetto (2016) to networks of incrementally input-to-state stable nonlinear control systems using the notion of approximate bisimulation relation. The recent result by Mallik, Schmuck, Soudjani, and Majumdar (2019) introduces a new system relation, called (approximate) disturbance bisimulation relation, as the basis for the compositional construction of symbolic models. Note that the proposed results by Tazaki and Imura (2008), Pola et al. (2016), and Mallik et al. (2019) use the small-gain type conditions and provide complete symbolic models of interconnected systems compositionally. The recent results by Swikir, Girard, and Zamani (2018) introduce different conditions to handle the compositional construction of complete finite abstractions by leveraging techniques from dissipativity theory (Arcak, Meissen, \& Packard, 2016). There are also other results in the literature (Hussein, Ames, \& Tabuada, 2017; Kim, Arcak, \& Zamani, 2018; Meyer, Girard, \& Witrant, 2018) which provide sound symbolic models of interconnected systems, compositionally, without requiring any stability property or condition on the gains of subsystems.

In this work, we introduce a compositional approach for the construction of complete finite abstractions of interconnected nonlinear discrete-time control systems using more general small-gain type conditions. First, we introduce a notion of socalled alternating simulation functions inspired by Girard and Pappas (2009, Definition 1) as a system relation. Given alternating simulation functions between subsystems and their finite abstractions, we derive some small-gain type conditions to construct an overall alternating simulation function as a relation between the interconnected abstractions and the concrete network. In addition, we provide a framework for the construction of finite abstractions together with their corresponding alternating simulation functions for discrete-time control systems satisfying incremental input-to-state stabilizability property (Angeli, 2002). Finally, we illustrate our results by compositionally constructing finite abstractions of two networks of (linear and nonlinear) discrete-time control subsystems and their corresponding alternating simulation functions. These case studies particularly elucidate the effectiveness of the proposed results in comparison with the existing compositional result using dissipativity-type conditions by Swikir et al. (2018).

One can leverage the compositionally constructed finite abstractions here to synthesize controllers monolithically or also compositionally to achieve some high-level properties; see the result by Meyer et al. (2018) and references therein. In particular, once finite abstractions are constructed for given concrete subsystems along with the corresponding alternating simulation functions, one can design local controllers also compositionally for those abstractions, and then refine them to the concrete subsystems provided that the given specification for the overall network is decomposable (see the first case study). Particularly, based on the assume-guarantee reasoning approach (Henzinger, Shaz, \& Rajamani, 1998), the local controllers are synthesized by assuming that the other subsystems meet their local specifications.

Related work. Results by Mallik et al. (2019), Pola et al. (2016), and Tazaki and Imura (2008) use the small-gain type conditions (Mallik et al. (2019, condition (13)), Pola et al. (2016, condition $r\left(A_{k}^{-1} C_{k}\right)<1$, Theorem 1), and Tazaki and Imura (2008, condition (17))) to facilitate the compositional construction of complete finite abstractions. Unfortunately, those small-gain type conditions are conservative, in the sense that they are all formulated in terms of "almost" linear gains, which means the considered subsystems should have a (nearly) linear behavior. Those conditions may not hold in general for systems with nonlinear gain functions (cf. Remark 3.6 in the paper). Here, we introduce more general small-gain type compositional conditions formulated in a general nonlinear form which can be applied to both linear and nonlinear gain functions without making any preassumptions on them. In addition, assuming a fully connected network, in the proposed compositionality results by Mallik et al (2019), Pola et al. (2016), Swikir et al. (2018), and Tazaki and Imura (2008) the overall approximation error is either proportional to the summation of the approximation errors of finite abstractions of subsystems or lower bounded by the summation of positive and strictly increasing functions of quantization parameters of all subsystems. On the other hand, in the proposed results here the overall approximation error is proportional to the maximum of the approximation errors of finite abstractions of subsystems which are determined independently of the number of subsystems. Therefore, the results here can potentially provide complete finite abstractions for large-scale interconnected systems with much smaller approximation error in comparison with those proposed by Mallik et al. (2019), Pola et al. (2016), Swikir et al. (2018), and Tazaki and Imura (2008) (cf. case studies for a comparison with Swikir et al. (2018)).

\section{Notation and preliminaries}

\subsection{Notation}

We denote by $\mathbb{R}, \mathbb{Z}$, and $\mathbb{N}$ the set of real numbers, integers, and non-negative integers, respectively. These symbols are annotated with subscripts to restrict them in the obvious way, e.g., $\mathbb{R}_{>0}$ denotes the positive real numbers. We denote the closed, open, and half-open intervals in $\mathbb{R}$ by $[a, b],(a, b),[a, b)$, and $(a, b]$, respectively. For $a, b \in \mathbb{N}$ and $a \leq b$, we use $[a ; b],(a ; b)$, $[a ; b)$, and $(a ; b]$ to denote the corresponding intervals in $\mathbb{N}$. Given $N \in \mathbb{N}_{>1}$, vectors $v_{i} \in \mathbb{R}^{n_{i}}, n_{i} \in \mathbb{N}_{>1}$, and $i \in[1 ; N]$, we use $v=\left[v_{1} ; \ldots ; v_{N}\right]$ to denote the vector in $\mathbb{R}^{n}$ with $n=\sum_{i} n_{i}$ consisting of the concatenation of vectors $v_{i}$. Note that given any $v \in \mathbb{R}^{n}, v \geq 0$ if $v_{i} \geq 0$ for any $i \in[1 ; n]$. We denote the identity and zero matrix in $\mathbb{R}^{n \times n}$ by $I_{n}$ and $0_{n}$, respectively. The individual elements in a matrix $A \in \mathbb{R}^{m \times n}$, are denoted by $\{A\}_{i j}$, where $i \in[1 ; m]$ and $j \in[1 ; n]$. We denote by $\|\cdot\|$ and $\|\cdot\|_{2}$ the infinity and Euclidean norm, respectively. Given any $a \in \mathbb{R},|a|$ denotes the absolute value of $a$. Given sets $X$ and $Y$, we denote by $f: X \rightarrow Y$ an ordinary map of $X$ into $Y$, whereas $f: X \rightrightarrows Y$ denotes a set-valued map (Rockafellar \& Wets, 2009). Given a function $f: \mathbb{R}^{n} \rightarrow \mathbb{R}^{m}$ and $\bar{x} \in \mathbb{R}^{m}$, we use $f \equiv \bar{x}$ to denote that $f(x)=\bar{x}$ for all $x \in \mathbb{R}^{n}$. If $\bar{x}$ is the zero vector, we simply write $f \equiv 0$. We denote by $|\cdot|$ the cardinality of a given set and by $\varnothing$ the empty set. A set $S \subseteq \mathbb{R}^{n}$ is a finite union of boxes if $S=\bigcup_{j=1}^{M} S_{j}$ for some $M \in \mathbb{N}$, where $S_{j}=\prod_{i=1}^{n}\left[c_{i}^{j}, d_{i}^{j}\right] \subseteq \mathbb{R}^{n}$ with $c_{i}^{j}<d_{i}^{j}$. For any set $S \subseteq \mathbb{R}^{n}$ of the form of finite union of boxes, we define $[S]_{\eta}=\left\{a \in S \mid a_{i}=k_{i} \eta, k_{i} \in \mathbb{Z}, i=1, \ldots, n\right\}$, where $0<$ $\eta \leq \operatorname{span}(S), \operatorname{span}(S)=\min _{j=1, \ldots, M} \eta_{S_{j}}, \eta_{S_{j}}=\min \left\{\left|d_{1}^{j}-c_{1}^{j}\right|, \ldots\right.$, $\left.\left|d_{n}^{j}-c_{n}^{j}\right|\right\}$. With a slight abuse of notation, we use $[S]_{0}:=S$. The set $[S]_{\eta}$ will be used as a finite approximation of the set $S$ with precision $\eta>0$. Note that $[S]_{\eta} \neq \varnothing$ for any $\eta \leq \operatorname{span}(S)$. Given sets $S$ and $[S]_{\eta}, \vartheta_{\eta}: S \rightarrow[S]_{\eta}$ is an approximation map that assigns for any $x \in S$ a representative point $\hat{x} \in[S]_{\eta}$ such that 
$\|x-\hat{x}\|<\eta$. Given sets $U$ and $S \subset U$, the complement of $S$ with respect to $U$ is defined as $U \backslash S=\{x: x \in U, x \notin S\}$. We use notations $\mathcal{K}$ and $\mathcal{K}_{\infty}$ to denote different classes of comparison functions, as follows: $\mathcal{K}=\left\{\alpha: \mathbb{R}_{\geq 0} \rightarrow \mathbb{R}_{\geq 0} \mid \alpha\right.$ is continuous, strictly increasing, and $\alpha(0)=0\} ; \mathcal{K}_{\infty}=\left\{\alpha \in \mathcal{K} \mid \lim _{r \rightarrow \infty} \alpha(r)=\right.$ $\infty$ \}. For $\alpha, \gamma \in \mathcal{K}_{\infty}$ we write $\alpha<\gamma$ if $\alpha(s)<\gamma(s)$ for all $s>0$, and $\mathcal{I}_{d} \in \mathcal{K}_{\infty}$ denotes the identity function.

\subsection{Discrete-time control systems}

In this paper we study discrete-time control systems of the following form.

Definition 2.1. A discrete-time control system $\Sigma$ is defined by the tuple $\Sigma=(\mathbb{X}, \mathbb{U}, \mathbb{W}, \mathcal{U}, \mathcal{W}, f, \mathbb{Y}, h)$, where $\mathbb{X}, \mathbb{U}, \mathbb{W}$, and $\mathbb{Y}$ are the state set, external input set, internal input set, and output set, respectively, and are assumed to be subsets of normed vector spaces with appropriate finite dimensions. Sets $\mathcal{U}$ and $\mathcal{W}$ denote the set of all bounded input functions $v: \mathbb{N} \rightarrow \mathbb{U}$ and $\omega: \mathbb{N} \rightarrow \mathbb{W}$, respectively. The set-valued map $f: \mathbb{X} \times \mathbb{U} \times \mathbb{W} \rightrightarrows \mathbb{X}$ is called the transition function, and $h: \mathbb{X} \rightarrow \mathbb{Y}$ is the output map. The discrete-time control system $\Sigma$ is described by difference inclusions of the form

$\Sigma:\left\{\begin{aligned} \mathbf{x}(k+1) & \in f(\mathbf{x}(k), v(k), \omega(k)), \\ \mathbf{y}(k) & =h(\mathbf{x}(k)),\end{aligned}\right.$

where $\mathbf{x}: \mathbb{N} \rightarrow \mathbb{X}, \mathbf{y}: \mathbb{N} \rightarrow \mathbb{Y}, v \in \mathcal{U}$, and $\omega \in \mathcal{W}$ are the state signal, output signal, external input signal, and internal input signal, respectively.

System $\Sigma=(\mathbb{X}, \mathbb{U}, \mathbb{W}, \mathcal{U}, \mathcal{W}, f, \mathbb{Y}, h)$ is called deterministic if $|f(x, u, w)| \leq 1 \forall x \in \mathbb{X}, \forall u \in \mathbb{U}, \forall w \in \mathbb{W}$, and non-deterministic otherwise. System $\Sigma$ is called blocking if $\exists x \in \mathbb{X}, \forall u \in \mathbb{U}, \forall w \in$ $\mathbb{W}$ where $|f(x, u, w)|=0$ and non-blocking if $|f(x, u, w)| \neq 0$ $\forall x \in \mathbb{X}, \exists u \in \mathbb{U}, \exists w \in \mathbb{W}$. System $\Sigma$ is called finite if $\mathbb{X}, \mathbb{U}, \mathbb{W}$ are finite sets and infinite otherwise. In this paper, we only deal with non-blocking systems.

Now, we introduce a notion of so-called alternating simulation functions, inspired by Girard and Pappas (2009, Definition 1), which quantifies the error between systems $\Sigma$ and $\hat{\Sigma}$ both with internal inputs.

Definition 2.2. Let $\Sigma=(\mathbb{X}, \mathbb{U}, \mathbb{W}, \mathcal{U}, \mathcal{W}, f, \mathbb{Y}, h)$ and $\hat{\Sigma}=$ $(\hat{\mathbb{X}}, \hat{\mathbb{U}}, \hat{\mathbb{W}}, \hat{\mathcal{U}}, \hat{\mathcal{W}}, \hat{f}, \hat{\mathbb{Y}}, \hat{h})$ where $\hat{\mathbb{W}} \subseteq \mathbb{W}$ and $\hat{\mathbb{Y}} \subseteq \mathbb{Y}$. A function $V: \mathbb{X} \times \hat{\mathbb{X}} \rightarrow \mathbb{R}_{\geq 0}$ is called an alternating simulation function from $\hat{\Sigma}$ to $\Sigma$ if $\forall x \in \mathbb{X}$ and $\forall \hat{x} \in \hat{\mathbb{X}}$, one has

$\alpha(\|h(x)-\hat{h}(\hat{x})\|) \leq V(x, \hat{x})$,

and $\forall x \in \mathbb{X}, \forall \hat{x} \in \hat{\mathbb{X}}, \forall \hat{u} \in \hat{\mathbb{U}}, \exists u \in \mathbb{U}, \forall w \in \mathbb{W}, \forall \hat{w} \in \hat{\mathbb{W}}$, $\forall x_{d} \in f(x, u, w), \exists \hat{x}_{d} \in \hat{f}(\hat{x}, \hat{u}, \hat{w})$ such that one gets

$$
\begin{aligned}
& V\left(x_{d}, \hat{x}_{d}\right) \\
& \quad \leq \max \left\{\sigma(V(x, \hat{x})), \rho_{\text {int }}(\|w-\hat{w}\|), \rho_{\text {ext }}(\|\hat{u}\|), \varepsilon\right\},
\end{aligned}
$$

for some $\alpha, \sigma, \rho_{\text {int }} \in \mathcal{K}_{\infty}$, where $\sigma<\mathcal{I}_{d}, \rho_{\text {ext }} \in \mathcal{K}_{\infty} \cup\{0\}$, and some $\varepsilon \in \mathbb{R}_{\geq 0}$.

Let us point out some differences between our notion of alternating simulation function and the one introduced by Girard and Pappas (2009). The notion of simulation function introduced by Girard and Pappas (2009, Definition 1) is defined between two continuous-time control systems, whereas in Definition 2.2, we define the alternating simulation function between two discrete-time control systems. Moreover, there is no distinction between internal and external inputs in the definition of simulation function introduced by Girard and Pappas (2009, Definition 1), whereas their distinctions in our work play a major role in providing the compositionality results later in the paper. Additionally, on the right-hand-side of (2.3), we introduce constant $\varepsilon \in \mathbb{R}_{>0}$ to allow the relation to be defined between two (in)finite systems. The role of this constant will become clear in Section 4 where we introduce finite systems. Such a constant does not appear in the definition of simulation function proposed by Girard and Pappas (2009, Definition 1) which makes it only suitable for infinite systems. Furthermore, we formulate the decay condition (2.3) in a max-form, while the decay condition proposed by Girard and Pappas (2009) is formulated in an implication-form.

If $\Sigma$ does not have internal inputs, which is the case for interconnected systems (cf. Definition 3.1), Definition 2.1 reduces to the tuple $\Sigma=(\mathbb{X}, \mathbb{U}, \mathcal{U}, f, \mathbb{Y}, h)$ and the set-valued map $f$ becomes $f: \mathbb{X} \times \mathbb{U} \rightrightarrows \mathbb{X}$. Correspondingly, (2.1) reduces to:

$\Sigma:\left\{\begin{array}{c}\mathbf{x}(k+1) \in f(\mathbf{x}(k), v(k)), \\ \mathbf{y}(k)=h(\mathbf{x}(k)) .\end{array}\right.$

Moreover, Definition 2.2 reduces to the following definition.

Definition 2.3. Consider systems $\Sigma=(\mathbb{X}, \mathbb{U}, \mathcal{U}, f, \mathbb{Y}, h)$ and $\hat{\Sigma}=(\hat{\mathbb{X}}, \hat{\mathbb{U}}, \hat{\mathcal{U}}, \hat{f}, \hat{\mathbb{Y}}, \hat{h})$, where $\hat{\mathbb{Y}} \subseteq \mathbb{Y}$. A function $\tilde{V}: \mathbb{X} \times \hat{\mathbb{X}} \rightarrow \mathbb{R}_{\geq 0}$ is called an alternating simulation function from $\hat{\Sigma}$ to $\Sigma$ if $\forall x \in \mathbb{X}$ and $\forall \hat{x} \in \hat{\mathbb{X}}$, one has

$\tilde{\alpha}(\|h(x)-\hat{h}(\hat{x})\|) \leq \tilde{V}(x, \hat{x})$,

and $\forall x \in \mathbb{X}, \forall \hat{x} \in \hat{\mathbb{X}}, \forall \hat{u} \in \hat{\mathbb{U}}, \exists u \in \mathbb{U}, \forall x_{d} \in f(x, u), \exists \hat{x}_{d} \in \hat{f}(\hat{x}, \hat{u})$ such that one gets

$\tilde{V}\left(x_{d}, \hat{x}_{d}\right) \leq \max \left\{\tilde{\sigma}(\tilde{V}(x, \hat{x})), \tilde{\rho}_{e x t}(\|\hat{u}\|), \tilde{\varepsilon}\right\}$,

for some $\tilde{\alpha}, \tilde{\sigma} \in \mathcal{K}_{\infty}$, where $\tilde{\sigma}<\mathcal{I}_{d}, \tilde{\rho}_{\text {ext }} \in \mathcal{K}_{\infty} \cup\{0\}$, and some $\tilde{\varepsilon} \in \mathbb{R}_{>0}$.

We say that a system $\hat{\Sigma}$ is approximately alternatingly simulated by a system $\Sigma$ or a system $\Sigma$ approximately alternatingly simulates a system $\hat{\Sigma}$, denoted by $\hat{\Sigma} \preceq \mathcal{A S} \Sigma$, if there exists an alternating simulation function from $\hat{\Sigma}$ to $\Sigma$ as in Definition 2.3.

We refer the interested readers to Pola and Tabuada (2009, Section 3.2) justifying in details the role of different quantifiers appeared before condition (2.6) in Definition 2.3 (condition (2.3) in Definition 2.2). In brief, those quantifiers capture the different role played by control inputs as well as nondeterminism in the system.

The next result shows that the existence of an alternating simulation function for systems without internal inputs implies the existence of an approximate alternating simulation relation between them as defined by Tabuada (2009).

Proposition 2.4. Consider systems $\Sigma=(\mathbb{X}, \mathbb{U}, \mathcal{U}, f, \mathbb{Y}, h)$ and $\hat{\Sigma}=(\hat{\mathbb{X}}, \hat{\mathbb{U}}, \hat{\mathcal{U}}, \hat{f}, \hat{\mathbb{Y}}, \hat{h})$, where $\hat{\mathbb{Y}} \subseteq \mathbb{Y}$. Assume $\tilde{V}$ is an alternating simulation function from $\hat{\Sigma}$ to $\Sigma$ as in Definition 2.3 and that there exists $v \in \mathbb{R}_{>0}$ such that $\|\hat{u}\| \leq v \forall \hat{u} \in \hat{\mathbb{U}}$. Then, relation $R \subseteq \mathbb{X} \times \hat{\mathbb{X}}$ defined by

$R=\left\{(x, \hat{x}) \in \mathbb{X} \times \hat{\mathbb{X}} \mid \tilde{V}(x, \hat{x}) \leq \max \left\{\tilde{\rho}_{\text {ext }}(v), \tilde{\varepsilon}\right\}\right\}$

is an $\hat{\varepsilon}$-approximate alternating simulation relation, defined by Tabuada (2009), from $\hat{\Sigma}$ to $\Sigma$ with

$\hat{\varepsilon}=\tilde{\alpha}^{-1}\left(\max \left\{\tilde{\rho}_{\text {ext }}(v), \tilde{\varepsilon}\right\}\right)$.

Proof. The proof consists of showing that $(i) \forall(x, \hat{x}) \in R$ we have $\|h(x)-\hat{h}(\hat{x})\| \leq \hat{\varepsilon}$; (ii) $\forall(x, \hat{x}) \in R$ and $\forall \hat{u} \in \hat{\mathbb{U}}, \exists u \in \mathbb{U}$, such that $\forall x_{d} \in f(x, u), \exists \hat{x}_{d} \in \hat{f}(\hat{x}, \hat{u})$ satisfying $\left(x_{d}, \hat{x}_{d}\right) \in R$. The first item is a simple consequence of the definition of $R$ and condition (2.5) (i.e. $\left.\tilde{\alpha}(\|h(x)-\hat{h}(\hat{x})\|) \leq \tilde{V}(x, \hat{x}) \leq \max \left\{\tilde{\rho}_{\text {ext }}(v), \tilde{\varepsilon}\right\}\right)$, which results in 
$\|h(x)-\hat{h}(\hat{x})\| \leq \tilde{\alpha}^{-1}\left(\max \left\{\tilde{\rho}_{\text {ext }}(v), \tilde{\varepsilon}\right\}\right)=\hat{\varepsilon}$. The second item follows immediately from the definition of $R$, condition (2.6), and the fact that $\tilde{\sigma}<\mathcal{I}_{d}$. In particular, we have $\tilde{V}\left(x_{d}, \hat{x}_{d}\right) \leq \max \left\{\tilde{\rho}_{\text {ext }}(v), \tilde{\varepsilon}\right\}$ which implies $\left(x_{d}, \hat{x}_{d}\right) \in R$.

\section{Compositionality result}

In this section, we analyze networks of discrete-time control subsystems and drive a general small-gain type condition under which we can construct an alternating simulation function from a network of abstractions to the concrete network by using alternating simulation functions of the subsystems. The definition of the network of discrete-time control subsystems is based on the notion of interconnected systems described by Tazaki and Imura (2008).

\subsection{Interconnected control systems}

We consider $N \in \mathbb{N}_{\geq 1}$ original control subsystems $\Sigma_{i}=$ $\left(\mathbb{X}_{i}, \mathbb{U}_{i}, \mathbb{W}_{i}, \mathcal{U}_{i}, \mathcal{W}_{i}, f_{i}, \mathbb{Y}_{i}, h_{i}\right), i \in[1 ; N]$, with partitioned internal inputs as

$w_{i}=\left[w_{i 1} ; \ldots ; w_{i(i-1)} ; w_{i(i+1)} ; \ldots ; w_{i N}\right]$,

$\mathbb{W}_{i}=\prod_{j=1}^{N-1} \mathbb{W}_{i j}$

with output map and set partitioned as

$$
\begin{aligned}
h_{i}\left(x_{i}\right) & =\left[h_{i 1}\left(x_{i}\right) ; \ldots ; h_{i N}\left(x_{i}\right)\right], \\
\mathbb{Y}_{i} & =\prod_{j=1}^{N} \mathbb{Y}_{i j} .
\end{aligned}
$$

We interpret the outputs $y_{i i}$ as external ones, whereas $y_{i j}$ with $i \neq$ $j$ are internal ones which are used to define the interconnected systems. In particular, we assume that the dimension of vector $w_{i j}$ is equal to that of vector $y_{j i}$. If there is no connection from subsystem $\Sigma_{i}$ to $\Sigma_{j}$, we set $h_{i j} \equiv 0$. Now, we define the notions of interconnections for control subsystems.

Definition 3.1. Consider $N \in \mathbb{N}_{\geq 1}$ control subsystems $\Sigma_{i}=$ $\left(\mathbb{X}_{i}, \mathbb{U}_{i}, \mathbb{W}_{i}, \mathcal{U}_{i}, \mathcal{W}_{i}, f_{i}, \mathbb{Y}_{i}, h_{i}\right), i \in[1 ; N]$, with the input-output structure given by (3.1)-(3.4). The interconnected control system $\Sigma=(\mathbb{X}, \mathbb{U}, \mathcal{U}, f, \mathbb{Y}, h)$, denoted by $\mathcal{I}_{\mathcal{M}}\left(\Sigma_{1}, \ldots, \Sigma_{N}\right)$, where $\mathcal{M} \in$ $\mathbb{R}^{N \times N}$ is a matrix with elements $\{\mathcal{M}\}_{i i}=0,\{\mathcal{M}\}_{i j}=\varpi_{i j}, \forall i, j \in$ $[1 ; N], i \neq j, 0 \leq \varpi_{i j} \leq \operatorname{span}\left(\mathbb{Y}_{j i}\right)$, is defined by $\mathbb{X}=\prod_{i=1}^{N} \mathbb{X}_{i}$, $\mathbb{U}=\prod_{i=1}^{N} \mathbb{U}_{i}, \mathcal{U}=\prod_{i=1}^{N} \mathcal{U}_{i}, \mathbb{Y}=\prod_{i=1}^{N} \mathbb{Y}_{i i}$, and maps

$f(x, u):=\left\{\left[x_{d 1} ; \ldots ; x_{d N}\right] \mid x_{d i} \in f_{i}\left(x_{i}, u_{i}, w_{i}\right) \forall i \in[1 ; N]\right\}$,

$h(x):=\left[h_{11}\left(x_{1}\right) ; \ldots ; h_{N N}\left(x_{N}\right)\right]$,

where $u=\left[u_{1} ; \ldots ; u_{N}\right], x=\left[x_{1} ; \ldots ; x_{N}\right]$, and subject to the constraint:

$w_{i j}=\vartheta_{\varpi_{i j}}\left(y_{j i}\right), \quad\left[\mathbb{Y}_{j i}\right]_{\varpi_{i j}} \subseteq \mathbb{W}_{i j}, \forall i, j \in[1 ; N], i \neq j$.

In the above definition, whenever $\varpi_{i j} \neq 0$, the sets $\mathbb{Y}_{j i}, \forall i, j \in$ $[1 ; N], i \neq j$, are assumed to be finite unions of boxes.

An example of an interconnection of three control subsystems $\Sigma_{1}, \Sigma_{2}$, and $\Sigma_{3}$ is illustrated in Fig. 1.

The following technical lemmas are used to prove some of the results in the next subsections.

Lemma 3.2. For any $a, b \in \mathbb{R}_{>0}$, the following holds

$a+b \leq \max \left\{\left(\mathcal{I}_{d}+\lambda\right)(a),\left(\mathcal{I}_{d}+\lambda^{-1}\right)(b)\right\}$,

for any $\lambda \in \mathcal{K}_{\infty}$.

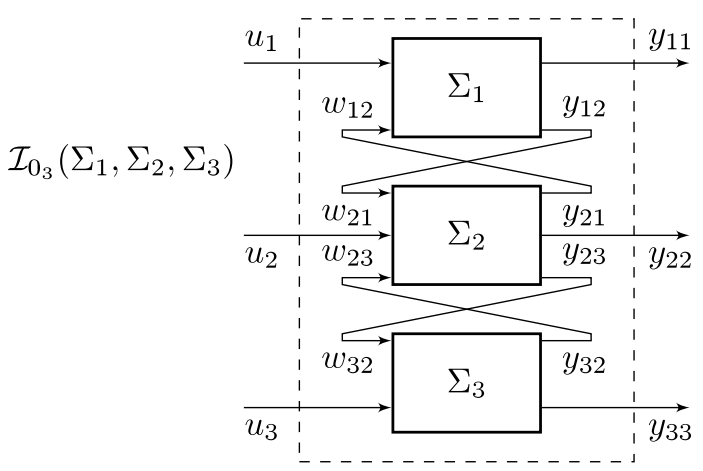

Fig. 1. Interconnection of three control subsystems $\Sigma_{1}, \Sigma_{2}$, and $\Sigma_{2}$ with $h_{13}=$ $h_{31}=0$.

Proof. Define $c=\lambda^{-1}(b)$. Now, one has

$a+b= \begin{cases}a+\lambda(c) \leq c+\lambda(c)=\left(\mathcal{I}_{d}+\lambda^{-1}\right)(b) & \text { if } a \leq c, \\ a+\lambda(c)<a+\lambda(a)=\left(\mathcal{I}_{d}+\lambda\right)(a) & \text { if } a>c,\end{cases}$

which implies (3.5).

The next lemma is borrowed from Kellett (2014).

Lemma 3.3. Consider $\alpha \in \mathcal{K}$ and $\chi \in \mathcal{K}_{\infty}$, where $\left(\chi-\mathcal{I}_{d}\right) \in \mathcal{K}_{\infty}$. Then for any $a, b \in \mathbb{R}_{\geq 0}$

$\alpha(a+b) \leq \alpha \circ \chi(a)+\alpha \circ \chi \circ\left(\chi-\mathcal{I}_{d}\right)^{-1}(b)$.

Next subsection provides one of the main results of the paper on the compositional construction of abstractions for networks of systems.

\subsection{Compositional construction of abstractions}

In this subsection, we assume that we are given $N$ original control subsystems $\Sigma=\left(\mathbb{X}_{i}, \mathbb{U}_{i}, \mathbb{W}_{i}, \mathcal{U}_{i}, \mathcal{W}_{i}, f_{i}, \mathbb{Y}_{i}, h_{i}\right)$ together with their corresponding abstractions $\hat{\Sigma}_{i}=\left(\hat{\mathbb{X}}_{i}, \hat{\mathbb{U}}_{i}, \hat{\mathbb{W}}_{i}, \hat{\mathcal{U}}_{i}, \hat{\mathcal{W}}_{i}, \hat{f}_{i}, \hat{\mathbb{Y}}_{i}\right.$, $\hat{h}_{i}$ ) and alternating simulation functions $V_{i}$ from $\hat{\Sigma}_{i}$ to $\Sigma_{i}$. Moreover, for functions $\sigma_{i}, \alpha_{i}$, and $\rho_{\text {int }}$ associated with $V_{i}, \forall i \in[1 ; N]$, appeared in Definition 2.2, we define

$\gamma_{i i}:=\sigma_{i}, \gamma_{i j}:=\left(\mathcal{I}_{d}+\lambda\right) \circ \rho_{\text {iint }} \circ \chi \circ \alpha_{j}^{-1}$,

$\forall j \in[1 ; N], j \neq i$, with arbitrarily chosen $\lambda, \chi \in \mathcal{K}_{\infty}$ with $\left(\chi-\mathcal{I}_{d}\right) \in \mathcal{K}_{\infty}$. Additionally, Let $\hat{\mathcal{M}} \in \mathbb{R}^{N \times N}$ be a matrix with elements $\{\hat{\mathcal{M}}\}_{i i}=0,\{\hat{\mathcal{M}}\}_{i j}=\hat{\varpi}_{i j}, \forall i, j \in[1 ; N], i \neq j, 0 \leq \hat{\varpi}_{i j} \leq$ $\operatorname{span}\left(\hat{\mathbb{Y}}_{j i}\right)$.

The next theorem provides a compositional approach on the construction of abstractions of networks of control subsystems and that of the corresponding alternating simulation functions.

Theorem 3.4. Consider the interconnected control system $\Sigma=$ $\mathcal{I}_{0_{N}}\left(\Sigma_{1}, \ldots, \Sigma_{N}\right)$ induced by $N \in \mathbb{N}_{\geq 1}$ control subsystems $\Sigma_{i}$. Assume that each $\Sigma_{i}$ and its abstraction $\hat{\Sigma}_{i}$ admit an alternating simulation function $V_{i}$. Let the following holds:

$\gamma_{i_{1} i_{2}} \circ \gamma_{i_{2} i_{3}} \circ \cdots \circ \gamma_{i_{r-1} i_{r}} \circ \gamma_{i_{r} i_{1}}<\mathcal{I}_{d}$,

$\forall\left(i_{1}, \ldots, i_{r}\right) \in\{1, \ldots, N\}^{r} \backslash\left\{\{1\}^{r}, \ldots,\{N\}^{r}\right\}$, where $r \in\{2, \ldots, N\}$. Then, there exist $\delta_{i} \in \mathcal{K}_{\infty}$ such that

$\tilde{V}(x, \hat{x}):=\max _{i}\left\{\delta_{i}^{-1} \circ V_{i}\left(x_{i}, \hat{x}_{i}\right)\right\}$

is an alternating simulation function from $\hat{\Sigma}=\mathcal{I}_{\hat{\mathcal{M}}}\left(\hat{\Sigma}_{1}, \ldots, \hat{\Sigma}_{N}\right)$ to $\Sigma$. 
Proof. Note that by using Theorem 5.2 introduced by Dashkovskiy, Rüffer, and Wirth (2010), condition (3.7) implies that $\exists \delta_{i} \in \mathcal{K}_{\infty} \forall i \in[1 ; N]$, satisfying

$\max _{j \in[1 ; N]}\left\{\delta_{i}^{-1} \circ \gamma_{i j} \circ \delta_{j}\right\}<\mathcal{I}_{d}$.

Now, we show that (2.5) holds for some $\mathcal{K}_{\infty}$ function $\tilde{\alpha}$. Consider any $x_{i} \in \mathbb{X}_{i}, \hat{x}_{i} \in \hat{\mathbb{X}}_{i}, \forall i \in[1 ; N]$. Then, one gets

$$
\begin{aligned}
\|h(x)-\hat{h}(\hat{x})\| & =\max _{i}\left\{\left\|h_{i i}\left(x_{i}\right)-\hat{h}_{i i}\left(\hat{x}_{i}\right)\right\|\right\} \\
& \leq \max _{i}\left\{\left\|h_{i}\left(x_{i}\right)-\hat{h}_{i}\left(\hat{x}_{i}\right)\right\|\right\} \\
& \leq \max _{i}\left\{\alpha_{i}^{-1} \circ V_{i}\left(x_{i}, \hat{x}_{i}\right)\right\} \\
& \leq \hat{\alpha} \circ \max _{i}\left\{\delta_{i}^{-1} \circ V_{i}\left(x_{i}, \hat{x}_{i}\right)\right\},
\end{aligned}
$$

where $\hat{\alpha}(s)=\max _{i}\left\{\alpha_{i}^{-1} \circ \delta_{i}(s)\right\}$ for all $s \in \mathbb{R}_{\geq 0}$. By defining $\tilde{\alpha}=\hat{\alpha}^{-1}$, one obtains

$\tilde{\alpha}(\|h(x)-\hat{h}(\hat{x})\|) \leq \tilde{V}(x, \hat{x})$

satisfying (2.5). Now, we show that (2.6) holds. Consider any $x=\left[x_{1} ; \ldots ; x_{N}\right] \in \mathbb{X}, \hat{x}=\left[\hat{x}_{1} ; \ldots ; \hat{x}_{N}\right] \in \hat{\mathbb{X}}$, and any $\hat{u}=$ $\left[\hat{u}_{1} ; \ldots ; \hat{u}_{N}\right] \in \hat{\mathbb{U}}$. For any $i \in[1 ; N]$, there exists $u_{i} \in \mathbb{U}_{i}$, consequently, a vector $u=\left[u_{1} ; \ldots ; u_{N}\right] \in \mathbb{U}$ such that for any $x_{d} \in f(x, u)$ there exists $\hat{x}_{d} \in \hat{f}(\hat{x}, \hat{u})$ satisfying (2.3) for each pair of subsystems $\Sigma_{i}$ and $\hat{\Sigma}_{i}$ with the internal inputs given by $\hat{w}_{i j}=\vartheta_{\hat{w}_{i j}}\left(\hat{y}_{j i}\right) \forall i, j \in[1 ; N], j \neq i$. One gets the chain of inequalities in (3.9) for some arbitrarily chosen $\lambda, \chi \in \mathcal{K}_{\infty}$ with $\left(\chi-\mathcal{I}_{d}\right) \in \mathcal{K}_{\infty}$. Observe that, in inequalities (3.9) (given in Box I ), we used Lemma 3.3 to go from line 7 to 8 , and Lemma 3.2 to go from line 9 to 10 . Define $\tilde{\sigma}, \tilde{\varepsilon}$, and $\tilde{\rho}_{\text {ext }}$ as follows:

$$
\begin{aligned}
\tilde{\sigma} & :=\max _{i, j}\left\{\delta_{i}^{-1} \circ \gamma_{i j} \circ \delta_{j}\right\}, \tilde{\varepsilon}:=\max _{i}\left\{\delta_{i}^{-1}\left(\phi_{i}\right)\right\}, \\
\tilde{\rho}_{\text {ext }}(s) & :=\left\{\begin{array}{l}
\max _{i}\left\{\delta_{i}^{-1} \circ \rho_{\text {iext }}\left(s_{i}\right)\right\} \\
\text { s.t. } \quad s=\left\|\left[s_{1}, \ldots, s_{n}\right]\right\|, s_{i} \geq 0,
\end{array}\right.
\end{aligned}
$$

where, $\forall i, j \in[1 ; N]$,

$\phi_{i}=\left(\mathcal{I}_{d}+\lambda^{-1}\right) \circ\left(\rho_{\text {iint }} \circ \chi \circ\left(\chi-\mathcal{I}_{d}\right)^{-1}\left(\max _{j, j \neq i}\left\{\hat{\omega}_{i j}\right\}\right)+\varepsilon_{i}\right)$

Observe that it follows from (3.8) that $\tilde{\sigma}<\mathcal{I}_{d}$. Then, one has

$\tilde{V}\left(x_{d}, \hat{x}_{d}\right) \leq \max \left\{\tilde{\sigma} \circ \tilde{V}(x, \hat{x}), \tilde{\rho}_{\text {ext }}(\|\hat{u}\|), \tilde{\varepsilon}\right\}$,

which satisfies (2.6), and implies that $\tilde{V}$ is indeed an alternating simulation function from $\hat{\Sigma}$ to $\Sigma$.

Note that, similar technique was proposed by Rungger and Zamani (2018) using nonlinear small-gain type condition to construct compositionally an approximate infinite abstraction of an interconnected continuous-time control system. Since in Rungger and Zamani (2018, Definition 2) a simulation function between each subsystem and its abstraction is formulated in a dissipativeform (Noroozi, Geiselhart, Grüne, Rüffer, \& Wirth, 2018), an extra operator (the operator $D$ in Rungger and Zamani (2018, equation $(12))$ ) is required to formulate the small-gain condition and to construct what is called an $\Omega$-path (Dashkovskiy et al., 2010, Definition 5.1), which is exactly the $\mathcal{K}_{\infty}$ functions $\delta_{i}, i \in N$, that satisfy condition (3.8) in our work. However, the definition of the simulation function in our work is formulated in a maxform (Noroozi et al., 2018) which results in not only simpler formulation of the small-gain condition but also the $\Omega$-path construction can be achieved without the need of the extra operator; see Dashkovskiy et al. (2010, Section 8.4).

Remark 3.5. Note that if, $\forall i \in[1 ; N], \rho_{\text {iint }}$ are linear functions, i.e., $\rho_{\text {iint }}(a+b)=\rho_{\text {iint }}(a)+\rho_{\text {iint }}(b), \forall a, b \in \mathbb{R}_{\geq 0}$, we omit the $\mathcal{K}_{\infty}$ function $\chi$ in (3.6) and (3.10); hence, $\gamma_{i j}$ and $\phi_{i}$ in the previous theorem reduce to $\gamma_{i j}=\left(\mathcal{I}_{d}+\lambda\right) \circ \rho_{\text {iint }} \circ \alpha_{j}^{-1}$ and $\phi_{i}=\left(\mathcal{I}_{d}+\lambda^{-1}\right) \circ\left(\rho_{\text {iint }} \circ\left(\max _{j, j \neq i}\left\{\hat{\varpi}_{i j}\right\}\right)+\varepsilon_{i}\right), \forall i, j \in[1 ; N], j \neq i$, respectively. Moreover, if $\hat{\varpi}_{i j}=0$, we omit the $\mathcal{K}_{\infty}$ function $\lambda$ in (3.6) and (3.10). Therefore, $\gamma_{i j}$ and $\phi_{i}$ reduce to $\gamma_{i j}=\rho_{i i n t} \circ \alpha_{j}^{-1}$ and $\phi_{i}=\varepsilon_{i}, \forall i, j \in[1 ; N], j \neq i$, respectively.

Remark 3.6. We emphasize that the proposed small-gain type condition in (3.7) is much more general than the ones proposed by Mallik et al. (2019) and Pola et al. (2016). To be more specific, consider the following system:

$\Sigma:\left\{\begin{array}{l}\mathbf{x}_{1}(k+1)=a_{1} \mathbf{x}_{1}(k)+b_{1} \sqrt{\left|\mathbf{x}_{2}(k)\right|}, \\ \mathbf{x}_{2}(k+1)=a_{2} \mathbf{x}_{2}(k)+b_{2} g\left(x_{1}(k)\right),\end{array}\right.$

where $0<a_{1}<1,0<a_{2}<1$, and function $g$ satisfies the following quadratic Lipschitz assumption: there exists an $L \in \mathbb{R}_{>0}$ such that: $\left|g(x)-g\left(x^{\prime}\right)\right| \leq L\left|x-x^{\prime}\right|^{2}$ for all $x, x^{\prime} \in \mathbb{R}$. One can easily verify that functions $V_{1}\left(x_{1}, \hat{x}_{1}\right)=\left|x_{1}-\hat{x}_{1}\right|$ and $V_{2}\left(x_{2}, \hat{x}_{2}\right)=$ $\left|x_{2}-\hat{x}_{2}\right|$ are alternating simulation functions from $\mathbf{x}_{1}$-subsystem to itself and $\mathbf{x}_{2}$-subsystem to itself, respectively. Here, one cannot come up with gain functions satisfying Assumption (A2) in Pola et al. (2016) globally (assumptions (1) and (2) in Mallik et al. (2019, Theorem 3) are continuous-time counterpart of Assumption (A2) in Pola et al. (2016)). In particular, those assumptions require existence of $\mathcal{K}_{\infty}$ functions being upper bounded by linear ones and lower bounded by quadratic ones which is impossible. On the other hand, the proposed small-gain condition (3.7) is still applicable here showing that $\tilde{V}(x, \hat{x}):=\max \left\{\delta_{1}^{-1} \circ V_{1}\left(x_{1}, \hat{x}_{1}\right), \delta_{2}^{-1} \circ\right.$ $\left.V_{2}\left(x_{2}, \hat{x}_{2}\right)\right\}$ is an alternating simulation function from $\Sigma$ to itself, for some appropriate $\delta_{1}, \delta_{2} \in \mathcal{K}_{\infty}$ satisfying (3.8) which is guaranteed to exist if $\left|b_{1}\right| \sqrt{\left|b_{2}\right| L}<1$ and $\left|b_{2}\right|\left(b_{1} L\right)^{2}<1$.

Remark 3.7. Here, we provide a general guideline on the computation of $\mathcal{K}_{\infty}$ functions $\delta_{i}, i \in[1 ; N]$ as the following: $(i)$ In a general case of having $N \geq 1$ subsystems, functions $\delta_{i}, i \in[1 ; N]$, can be constructed numerically using the algorithm proposed by Eaves (1972) and the technique provided by Dashkovskiy et al. (2010, Proposition 8.8), see Ruffer (2007, Chapter 4); (ii) Simple construction techniques are provided by Dashkovskiy et al. (2010, Section 9) and Jiang, Mareels, and Wang (1996) for the case of two and three subsystems, respectively; (iii) the $\mathcal{K}_{\infty}$ functions $\delta_{i}, i \in[1 ; N]$, can be always chosen as identity functions provided that $\gamma_{i j}<\mathcal{I}_{d}, \forall i, j \in[1 ; N]$, for functions $\gamma_{i j}$ appeared in (3.6).

\section{Construction of symbolic models}

In this section, we consider $\Sigma=(\mathbb{X}, \mathbb{U}, \mathbb{W}, \mathcal{U}, \mathcal{W}, f, \mathbb{Y}, h)$ as an infinite, deterministic control system and assume its output map $h$ satisfies the following general Lipschitz assumption: there exists an $\ell \in \mathcal{K}_{\infty}$ such that: $\left\|h(x)-h\left(x^{\prime}\right)\right\| \leq \ell\left(\left\|x-x^{\prime}\right\|\right)$ for all $x, x^{\prime} \in \mathbb{X}$. Note that this assumption on $h$ is not restrictive at all provided that one is interested to work on a compact subset of $\mathbb{X}$. In addition, the existence of an alternating simulation function between $\Sigma$ and its finite abstraction is established under the assumption that $\Sigma$ is so-called incrementally input-to-state stabilizable as defined next.

Definition 4.1. System $\Sigma=(\mathbb{X}, \mathbb{U}, \mathbb{W}, \mathcal{U}, \mathcal{W}, f, \mathbb{Y}, h)$ is called incrementally input-to-state stabilizable if there exist functions $\mathcal{H}: \mathbb{X} \rightarrow \mathbb{U}$ and $\mathcal{G}: \mathbb{X} \times \mathbb{X} \rightarrow \mathbb{R}_{\geq 0}$ such that $\forall x, x^{\prime} \in \mathbb{X}, \forall u, u^{\prime} \in \mathbb{U}$, $\forall w, w^{\prime} \in \mathbb{W}$, the inequalities:

$\underline{\alpha}\left(\left\|x-x^{\prime}\right\|\right) \leq \mathcal{G}\left(x, x^{\prime}\right) \leq \bar{\alpha}\left(\left\|x-x^{\prime}\right\|\right)$, 


$$
\begin{aligned}
& \tilde{V}\left(x_{d}, \hat{x}_{d}\right)=\max _{i}\left\{\delta_{i}^{-1} \circ V_{i}\left(x_{d_{i}}, \hat{x}_{d_{i}}\right)\right\} \\
& \leq \max _{i}\left\{\delta_{i}^{-1}\left(\max \left\{\sigma_{i} \circ V_{i}\left(x_{i}, \hat{x}_{i}\right), \rho_{\text {iint }}\left(\left\|w_{i}-\hat{w}_{i}\right\|\right), \rho_{\text {iext }}\left(\left\|\hat{u}_{i}\right\|\right), \varepsilon_{i}\right\}\right)\right\} \\
& =\max _{i}\left\{\delta_{i}^{-1}\left(\max \left\{\sigma_{i} \circ V_{i}\left(x_{i}, \hat{x}_{i}\right), \rho_{\text {iint }}\left(\max _{j, j \neq i}\left\{\left\|w_{i j}-\hat{w}_{i j}\right\|\right\}\right), \rho_{\text {iext }}\left(\left\|\hat{u}_{i}\right\|\right), \varepsilon_{i}\right\}\right)\right\} \\
& =\max _{i}\left\{\delta_{i}^{-1}\left(\max \left\{\sigma_{i} \circ V_{i}\left(x_{i}, \hat{x}_{i}\right), \rho_{i i n t}\left(\max _{j, j \neq i}\left\{\left\|y_{j i}-\vartheta_{\hat{\omega}_{i j}\left(\hat{y}_{j i}\right)}\right\|\right\}\right), \rho_{i e x t}\left(\left\|\hat{u}_{i}\right\|\right), \varepsilon_{i}\right\}\right)\right\} \\
& =\max _{i}\left\{\delta_{i}^{-1}\left(\max \left\{\sigma_{i} \circ V_{i}\left(x_{i}, \hat{x}_{i}\right), \rho_{i i n t}\left(\max _{j, j \neq i}\left\{\left\|y_{j i}-\hat{y}_{j i}+\hat{y}_{j i}-\vartheta_{\hat{\omega}_{i j}}\left(\hat{y}_{j i}\right)\right\|\right\}\right), \rho_{\text {iext }}\left(\left\|\hat{u}_{i}\right\|\right), \varepsilon_{i}\right\}\right)\right\} \\
& \leq \max _{i}\left\{\delta_{i}^{-1}\left(\max \left\{\sigma_{i} \circ V_{i}\left(x_{i}, \hat{x}_{i}\right), \rho_{i i n t}\left(\max _{j, j \neq i}\left\{\left\|h_{j}\left(x_{j}\right)-\hat{h}_{j}\left(\hat{x}_{j}\right)\right\|+\hat{\varpi}_{i j}\right\}\right), \rho_{i e x t}\left(\left\|\hat{u}_{i}\right\|\right), \varepsilon_{i}\right\}\right)\right\} \\
& \leq \max _{i}\left\{\delta_{i}^{-1}\left(\max \left\{\sigma_{i} \circ V_{i}\left(x_{i}, \hat{x}_{i}\right), \rho_{\text {iint }}\left(\max _{j, j \neq i}\left\{\alpha_{j}^{-1} \circ V_{j}\left(x_{j}, \hat{x}_{j}\right)+\hat{\omega}_{i j}\right\}\right), \rho_{\text {iext }}\left(\left\|\hat{u}_{i}\right\|\right), \varepsilon_{i}\right\}\right)\right\} \\
& \leq \max _{i}\left\{\delta _ { i } ^ { - 1 } \left(\operatorname { m a x } \left\{\sigma_{i} \circ V_{i}\left(x_{i}, \hat{x}_{i}\right), \rho_{\text {iint }} \circ \chi\left(\max _{j, j \neq i}\left\{\alpha_{j}^{-1} \circ V_{j}\left(x_{j}, \hat{x}_{j}\right)\right\}\right)\right.\right.\right. \\
& \left.\left.\left.+\rho_{\text {iint }} \circ \chi \circ\left(\chi-\mathcal{I}_{d}\right)^{-1}\left(\max _{j, j \neq i}\left\{\hat{\varpi}_{i j}\right\}\right), \rho_{\text {iext }}\left(\left\|\hat{u}_{i}\right\|\right), \varepsilon_{i}\right\}\right)\right\} \\
& \leq \max _{i}\left\{\delta _ { i } ^ { - 1 } \left(\operatorname { m a x } \left\{\sigma_{i} \circ V_{i}\left(x_{i}, \hat{x}_{i}\right), \rho_{\text {iint }} \circ \chi\left(\max _{j, j \neq i}\left\{\alpha_{j}^{-1} \circ V_{j}\left(x_{j}, \hat{x}_{j}\right)\right\}\right)\right.\right.\right. \\
& \left.\left.\left.+\rho_{\text {iint }} \circ \chi \circ\left(\chi-\mathcal{I}_{d}\right)^{-1}\left(\max _{j, j \neq i}\left\{\mu_{w i j}\right\}\right)+\varepsilon_{i}, \rho_{\text {iext }}\left(\left\|\hat{u}_{i}\right\|\right)\right\}\right)\right\} \\
& \leq \max _{i}\left\{\delta _ { i } ^ { - 1 } \left(\operatorname { m a x } \left\{\sigma_{i} \circ V_{i}\left(x_{i}, \hat{x}_{i}\right),\left(\mathcal{I}_{d}+\lambda\right) \circ \rho_{i i n t} \circ \chi\left(\max _{j, j \neq i}\left\{\alpha_{j}^{-1} \circ V_{j}\left(x_{j}, \hat{x}_{j}\right)\right\}\right),\right.\right.\right. \\
& \left.\left.\left.\rho_{\text {iext }}\left(\left\|\hat{u}_{i}\right\|\right),\left(\mathcal{I}_{d}+\lambda^{-1}\right) \circ\left(\rho_{\text {iint }} \circ \chi \circ\left(\chi-\mathcal{I}_{d}\right)^{-1}\left(\max _{j, j \neq i}\left\{\hat{\varpi}_{i j}\right\}\right)+\varepsilon_{i}\right)\right\}\right)\right\} \\
& \leq \max _{i, j}\left\{\delta_{i}^{-1}\left(\max \left\{\gamma_{i j} \circ V_{j}\left(x_{j}, \hat{x}_{j}\right), \rho_{\text {iext }}\left(\left\|\hat{u}_{i}\right\|\right), \phi_{i}\right\}\right)\right\} \\
& =\max _{i, j}\left\{\delta_{i}^{-1}\left(\max \left\{\gamma_{i j} \circ \delta_{j} \circ \delta_{j}^{-1} \circ V_{j}\left(x_{j}, \hat{x}_{j}\right), \rho_{i e x t}\left(\left\|\hat{u}_{i}\right\|\right), \phi_{i}\right\}\right)\right\} \\
& \leq \max _{i, j, l}\left\{\delta_{i}^{-1}\left(\max \left\{\gamma_{i j} \circ \delta_{j} \circ \delta_{l}^{-1} \circ V_{l}\left(x_{l}, \hat{x}_{l}\right), \rho_{i e x t}\left(\left\|\hat{u}_{i}\right\|\right), \phi_{i}\right\}\right)\right\} \\
& =\max _{i, j}\left\{\delta_{i}^{-1}\left(\max \left\{\gamma_{i j} \circ \delta_{j} \circ \tilde{V}(x, \hat{x}), \rho_{i e x t}\left(\left\|\hat{u}_{i}\right\|\right), \phi_{i}\right\}\right)\right\} \\
& =\max \left\{\tilde{\sigma} \circ \tilde{V}(x, \hat{x}), \max _{i}\left\{\delta_{i}^{-1} \circ \rho_{\text {iext }}\left(\left\|\hat{u}_{i}\right\|\right), \delta_{i}^{-1}\left(\phi_{i}\right)\right\}\right\},
\end{aligned}
$$

Box I.

and

$$
\begin{array}{r}
\mathcal{G}\left(f(x, \mathcal{H}(x)+u, w), f\left(x^{\prime}, \mathcal{H}\left(x^{\prime}\right)+u^{\prime}, w^{\prime}\right)\right)-\mathcal{G}\left(x, x^{\prime}\right) \\
\leq-\kappa\left(\mathcal{G}\left(x, x^{\prime}\right)\right)+\gamma_{i n t}\left(\left\|w-w^{\prime}\right\|\right)+\gamma_{e x t}\left(\left\|u-u^{\prime}\right\|\right)
\end{array}
$$

hold for some $\underline{\alpha}, \bar{\alpha}, \kappa, \gamma_{\text {int }}, \gamma_{\text {ext }} \in \mathcal{K}_{\infty}$.

Remark that in Definition 4.1, we implicitly assume that $\mathcal{H}(x)+$ $u \in \mathbb{U}$ for any $x \in \mathbb{X}$ and any $u \in \mathbb{U}$. Note that any classically stabilizable linear control system is also incrementally stabilizable as in Definition 4.1. For nonlinear control systems, the notion of incremental stabilizability as in Definition 4.1 is stronger than conventional stabilizability. We refer the interested readers to Tran, Rüffer, and Kellett (2016) for detailed information on incremental input-to-state stability of discrete-time control systems.

Now, we construct a finite abstraction $\hat{\Sigma}$ of an incrementally input-to-state stabilizable control system $\Sigma$ as the following.

Definition 4.2. Let $\Sigma=(\mathbb{X}, \mathbb{U}, \mathbb{W}, \mathcal{U}, \mathcal{W}, f, \mathbb{Y}, h)$ be incrementally input-to-state stabilizable as in Definition 4.1 , where
$\mathbb{X}, \mathbb{U}, \mathbb{W}$ are assumed to be finite unions of boxes. One can construct a finite system

$\hat{\Sigma}=(\hat{\mathbb{X}}, \hat{\mathbb{U}}, \hat{\mathbb{W}}, \hat{\mathcal{U}}, \hat{\mathcal{W}}, \hat{f}, \hat{\mathbb{Y}}, \hat{h})$,

where:

- $\hat{\mathbb{X}}=[\mathbb{X}]_{\eta}$, where $0<\eta \leq \operatorname{span}(\mathbb{X})$ is the state set quantization parameter;

- $\hat{\mathbb{U}}=[\mathbb{U}]_{\mu}$, where $0<\mu \leq \operatorname{span}(\mathbb{U})$ is the external input set quantization parameter;

- $\hat{\mathbb{W}}=[\mathbb{W}]_{\hat{\omega}}$, where $0 \leq \hat{\omega} \leq \operatorname{span}(\mathbb{W})$ is the internal input set quantization parameter;

- $\hat{x}_{d} \in \hat{f}(\hat{x}, \hat{u}, \hat{w})$ iff $\left\|\hat{x}_{d}-f(\hat{x}, \mathcal{H}(\hat{x})+\hat{u}, \hat{w})\right\| \leq \eta$;

- $\hat{\mathbb{Y}}=\{h(\hat{x}) \mid \hat{x} \in \hat{\mathbb{X}}\}$;

- $\hat{h}=h$.

Next, we establish the relation between $\Sigma$ and $\hat{\Sigma}$, introduced above, via the notion of alternating simulation function in Definition 2.2. In particulate, we show that $\hat{\Sigma}$ is a complete finite abstraction of $\Sigma$ by proving that function $\mathcal{G}$ in Definition 4.1 is an alternating simulation function from $\hat{\Sigma}$ to $\Sigma$ and from $\Sigma$ to $\hat{\Sigma}$. 
Theorem 4.3. Let $\Sigma$ be an incrementally input-to-state stabilizable control system as in Definition 4.1 and $\hat{\Sigma}$ be a finite system as constructed in Definition 4.2. Assume that there exists a function $\hat{\gamma} \in \mathcal{K}_{\infty}$ such that for any $x, x^{\prime}, x^{\prime \prime} \in \mathbb{X}$ one has

$\mathcal{G}\left(x, x^{\prime}\right) \leq \mathcal{G}\left(x, x^{\prime \prime}\right)+\hat{\gamma}\left(\left\|x^{\prime}-x^{\prime \prime}\right\|\right)$

for $\mathcal{G}$ as in Definition 4.1. Then $\mathcal{G}$ is actually an alternating simulation function from $\hat{\Sigma}$ to $\Sigma$ and from $\Sigma$ to $\hat{\Sigma}$.

Proof. Given the Lipschitz assumption on $h$ and since $\Sigma$ is incrementally input-to-state stabilizable, from (4.1), $\forall x \in \mathbb{X}$ and $\forall \hat{x} \in \hat{\mathbb{X}}$, we have

$\|h(x)-\hat{h}(\hat{x})\| \leq \ell(\|x-\hat{x}\|) \leq \hat{\alpha}(\mathcal{G}(x, \hat{x}))$,

where $\hat{\alpha}=\ell \circ \underline{\alpha}^{-1}$. By defining $\alpha=\hat{\alpha}^{-1}$, one obtains

$\alpha(\|h(x)-\hat{h}(\hat{x})\|) \leq \mathcal{G}(x, \hat{x})$,

satisfying (2.2). Now from (4.4), $\forall x \in \mathbb{X}, \forall \hat{x} \in \hat{\mathbb{X}}, \forall \hat{u} \in \hat{\mathbb{U}}, \forall w \in$ $\mathbb{W}, \forall \hat{w} \in \hat{\mathbb{W}}$, we have

$$
\begin{aligned}
\mathcal{G}(f(x, & \left.\mathcal{H}(x)+\hat{u}, w), \hat{x}_{d}\right) \\
\leq & \mathcal{G}(f(x, \mathcal{H}(x)+\hat{u}, w), f(\hat{x}, \mathcal{H}(\hat{x})+\hat{u}, \hat{w})) \\
& +\hat{\gamma}\left(\left\|\hat{x}_{d}-f(\hat{x}, \mathcal{H}(\hat{x})+\hat{u}, \hat{w})\right\|\right)
\end{aligned}
$$

for any $\hat{x}_{d} \in \hat{f}(\hat{x}, \hat{u}, \hat{w})$. Now, from Definition 4.2 , the above inequality reduces to

$$
\begin{aligned}
& \mathcal{G}\left(f(x, \mathcal{H}(x)+\hat{u}, w), \hat{x}_{d}\right) \\
& \quad \leq \mathcal{G}(f(x, \mathcal{H}(x)+\hat{u}, w), f(\hat{x}, \mathcal{H}(\hat{x})+\hat{u}, \hat{w}))+\hat{\gamma}(\eta) .
\end{aligned}
$$

Note that by (4.2), we get

$$
\begin{aligned}
& \mathcal{G}(f(x, \mathcal{H}(x)+\hat{u}, w), f(\hat{x}, \mathcal{H}(\hat{x})+\hat{u}, \hat{w}))-\mathcal{G}(x, \hat{x}) \\
& \quad \leq-\kappa(\mathcal{G}(x, \hat{x}))+\gamma_{i n t}(\|w-\hat{w}\|) .
\end{aligned}
$$

Hence, $\forall x \in \mathbb{X}, \forall \hat{x} \in \hat{\mathbb{X}}, \forall \hat{u} \in \hat{\mathbb{U}}$, and $\forall w \in \mathbb{W}, \forall \hat{w} \in \widehat{\mathbb{W}}$, one obtains

$$
\begin{aligned}
& \mathcal{G}\left(f(x, \mathcal{H}(x)+\hat{u}, w), \hat{x}_{d}\right)-\mathcal{G}(x, \hat{x}) \\
& \quad \leq-\kappa(\mathcal{G}(x, \hat{x}))+\gamma_{\text {int }}(\|w-\hat{w}\|)+\hat{\gamma}(\eta)
\end{aligned}
$$

for any $\hat{x}_{d} \in \hat{f}(\hat{x}, \hat{u}, \hat{w})$. Using the previous inequality and by following a similar argument as the one in the proof of Theorem 1 in Swikir et al. (2018), one obtains

$$
\begin{aligned}
& \mathcal{G}\left(f(x, \mathcal{H}(x)+\hat{u}, w), \hat{x}_{d}\right) \\
& \quad \leq \max \left\{\tilde{\kappa}(\mathcal{G}(x, \hat{x})), \tilde{\gamma}_{\text {int }}(\|w-\hat{w}\|), \tilde{\gamma}(\eta)\right\},
\end{aligned}
$$

where $\tilde{\kappa}=\mathcal{I}_{d}-\left(\mathcal{I}_{d}-\psi\right) \circ \hat{\kappa}, \tilde{\gamma}_{i n t}=\left(\mathcal{I}_{d}+\lambda\right) \circ \hat{\kappa}^{-1} \circ \psi^{-1} \circ$ $\chi \circ \gamma_{i n t}, \tilde{\gamma}=\left(\mathcal{I}_{d}+\lambda^{-1}\right) \circ \hat{\kappa}^{-1} \circ \psi^{-1} \circ \chi \circ\left(\chi-\mathcal{I}_{d}\right)^{-1} \circ \hat{\gamma}$, where $\lambda, \chi, \psi, \hat{\kappa}$ are some arbitrarily chosen $\mathcal{K}_{\infty}$ functions with $\mathcal{I}_{d}-\psi \in \mathcal{K}_{\infty}, \quad \chi-\mathcal{I}_{d} \in \mathcal{K}_{\infty}, \mathcal{I}_{d}-\hat{\kappa} \in \mathcal{K}_{\infty}$ and $\hat{\kappa} \leq \kappa$. Hence, inequality (2.3) is satisfied with $u=\mathcal{H}(x)+\hat{u}, \sigma=\tilde{\kappa}, \rho_{\text {int }}=\tilde{\gamma}_{\text {int }}$, $\rho_{\text {ext }}(s)=0 \forall s \in \mathbb{R}_{\geq 0}, \varepsilon=\tilde{\gamma}(\eta)$, and, hence, $\mathcal{G}$ is an alternating simulation function from $\hat{\Sigma}$ to $\Sigma$. Similarly, we can also show that $\mathcal{G}$ is an alternating simulation function from $\Sigma$ to $\hat{\Sigma}$. In particular, by the definition of $\hat{\mathbb{U}}$, for any $u=\mathcal{H}(x)+\tilde{u} \in \mathbb{U}$ there always exists $\hat{u} \in \hat{\mathbb{U}}$ such that $\gamma_{\text {ext }}(\|\tilde{u}-\hat{u}\|) \leq \gamma_{\text {ext }}(\mu)$ which results in $\varepsilon=\left(\mathcal{I}_{d}+\lambda^{-1}\right) \circ \hat{\kappa}^{-1} \circ \psi^{-1} \circ \chi \circ\left(\chi-\mathcal{I}_{d}\right)^{-1}\left(\gamma_{\text {ext }}(\mu)+\hat{\gamma}(\eta)\right)$. Other terms in the alternating simulation function $\mathcal{G}$ are the same as the first part of the proof.

Remark 4.4. Observe that if $\gamma_{\text {int }}$ and $\hat{\gamma}$ are linear functions in the previous theorem, $\tilde{\gamma}_{\text {int }}$ and $\tilde{\gamma}$ reduce to $\tilde{\gamma}_{\text {int }}=\left(\mathcal{I}_{d}+\lambda\right) \circ \hat{\kappa}^{-1} \circ$ $\psi^{-1} \circ \gamma_{\text {int }}$ and $\tilde{\gamma}=\left(\mathcal{I}_{d}+\lambda^{-1}\right) \circ \hat{\kappa}^{-1} \circ \psi^{-1} \circ \hat{\gamma}$, respectively.
Remark 4.5. Although the choices of $\mathcal{K}_{\infty}$ functions $\lambda, \chi, \psi$, and $\hat{\kappa}$ in the previous theorem mainly depend on the dynamic of the given control systems, we provide a general guideline on choosing those functions as follows: $(i)$ In order to reduce the undesirable effect of the inverse of $\hat{\kappa}$ and $\psi$ in satisfying the small-gain condition in (3.7), or in computing the value of the overall approximation error in (2.7), one should choose those functions to behave very close to the identity function; (ii) Regarding $\lambda$ and $\chi$, one should choose those functions such that the small gain condition in (3.7) is possibly satisfied, and then compute the overall approximation error in (2.7). If the computed error is acceptable by the user, no further action is required; otherwise one should start slightly modifying those functions until a smaller error is achieved while ensuring that the small gain condition is not violated. For example, one can scale the $\mathcal{K}_{\infty}$ function $\lambda$ by a linear function $\beta(s)=c s \in \mathcal{K}_{\infty}, \forall s \in \mathbb{R}_{\geq 0}, c>1$, and then, using $\beta \circ \lambda$ instead of $\lambda$, start increasing the value of $c$ until a smaller error is obtained. Same procedure can be simultaneously applied to the $\mathcal{K}_{\infty}$ function $\chi$. It may be the case that the desired error is not achievable with the chosen $\lambda$ and $\chi$, then one should start over and choose different $\lambda$ and $\chi$ and go through similar procedure again.

Remark that condition (4.4) is not restrictive at all provided that one is interested to work on a compact subset of $\mathbb{X}$. We refer the interested readers to the explanation provided after equation (V.2) in Zamani, Mohajerin Esfahani, Majumdar, Abate, and Lygeros (2014) on how to compute such function $\hat{\gamma}$.

Now we provide similar results as in the first part of this section but tailored to linear control systems which are computationally much more efficient.

\subsection{Discrete-time linear control systems}

The class of discrete-time linear control systems, considered in this subsection, is given by

$$
\Sigma:\left\{\begin{aligned}
x(k+1) & =A x(k)+B u(k)+D w(k), \\
y(k) & =C x(k),
\end{aligned}\right.
$$

where $A \in \mathbb{R}^{n \times n}, B \in \mathbb{R}^{n \times m}, D \in \mathbb{R}^{n \times p}, C \in \mathbb{R}^{q \times n}$. We use the tuple $\Sigma=(A, B, C, D)$ to refer to the class of control systems of the form (4.5). Remark that the incremental input-tostate stabilizability assumption in Definition 4.2 boils down in the linear case to the following assumption.

Assumption 4.6. Let $\Sigma=(A, B, C, D)$. Assume that there exist matrices $Z \succ 0$ and $K$ of appropriate dimensions such that the matrix inequality

$(1+2 \theta)(A+B K)^{T} Z(A+B K) \preceq \kappa_{c} Z$

holds for some constants $0<\kappa_{c}<1$, and $\theta \in \mathbb{R}_{>0}$.

Note that condition (4.6) is nothing more than pair $(A, B)$ being stabilizable (Antsaklis \& Michel, 2007).

Remark 4.7. Given constants $\kappa_{c}$ and $\theta$, one can easily see that inequality (4.6) is not jointly convex on decision variables $Z$ and $K$ and, hence, not amenable to existing semidefinite tools for linear matrix inequalities (LMI). However, using Schur complement, one can easily transform inequality (4.6) to the following LMI over decision variables $Q$ and $M$ :

$\left[\begin{array}{cc}-\kappa_{c} Q & Q A^{T}+M^{T} B^{T} \\ A Q+B M & -(1+2 \theta) Q\end{array}\right] \preceq 0, Q \succ 0$,

where $Q=Z^{-1}$ and $M=K Q$. 
Now, Theorem 4.3 reduces to the following one for linear systems.

Theorem 4.8. Consider $\Sigma=(A, B, C, D)$ and the finite abstraction $\hat{\Sigma}$ constructed as in Definition 4.2. Suppose Assumption 4.6 holds. Then, function

$V(x, \hat{x})=\sqrt{(x-\hat{x})^{T} Z(x-\hat{x})}$,

is an alternating simulation function from $\hat{\Sigma}$ to $\Sigma$ and from $\Sigma$ to $\hat{\Sigma}$.

Proof. First, we show that condition (2.2) holds. Since $C=\hat{C}$, we have

$\|C x-\hat{C} \hat{x}\| \leq \sqrt{n \lambda_{\max }\left(C^{T} C\right)}\|x-\hat{x}\|$,

and similarity

$\sqrt{\lambda_{\min }(Z)}\|x-\hat{x}\| \leq \sqrt{(x-\hat{x})^{T} Z(x-\hat{x})}$.

It can be readily verified that (2.2) holds for $V$ defined in (4.7) with $\alpha(s)=\sqrt{\frac{\lambda_{\min }(Z)}{n \lambda_{\max }\left(C^{T} C\right)}} s$ for any $s \in \mathbb{R}_{\geq 0}$. We continue to show that (2.3) holds as well. Let $x, \hat{x}, \hat{u}$, and $\hat{w}$ be given, and choose $u$ as $u:=K(x-\hat{x})+\hat{u}$. Let $x_{d}=A x+B u+D w$, and $\hat{x}_{d}$ be defined as in Definition 4.2. Define $F:=A \hat{x}+B \hat{u}+D \hat{w}-\hat{x}_{d}$, and $\hat{\kappa}_{c}:=1-\sqrt{\kappa_{c}}$. Now, one obtains the chain of inequalities (4.8) (given in Box II). By following a similar argument as the one in the proof of Theorem 1 in Swikir et al. (2018), one gets (4.9) (given in Box II) where $\tilde{\kappa}=\left(1-\hat{\kappa}_{c}\left(1-\psi_{c}\right)\right)$, satisfying (2.3) with $\sigma(s)=\tilde{\kappa} s, \rho_{\text {ext }}(s)=0, \rho_{\text {int }}(s)=\frac{\left(1+\delta_{c}\right)}{\hat{\kappa}_{c} \psi_{c}} \sqrt{p \frac{\left(1+\theta+\theta^{2}\right)}{\theta}}\|\sqrt{Z} D\|_{2} s$, $\forall s \in \mathbb{R}_{\geq 0}, \varepsilon=\frac{\left(1+1 / \delta_{c}\right)}{\hat{\kappa}_{c} \psi_{c}} \sqrt{\frac{n(2+\theta) \lambda_{\max }(Z)}{\theta}} \eta$, where $\psi_{c}$ and $\delta_{c}$ can be chosen arbitrarily such that $0<\psi_{c}<1$ and $\delta_{c}>0$. Hence, the proposed $V$ in (4.7) is an alternating simulation function from $\hat{\Sigma}$ to $\Sigma$. The rest of the proof follows similar argument. In particular, by the definition of $\hat{\mathbb{U}}$, for any $u=K(x-\hat{x})+\tilde{u} \in \mathbb{U}$ there always exists $\hat{u} \in \hat{\mathbb{U}}$ such that $\|B\|\|\tilde{u}-\hat{u}\| \leq\|B\| \mu$ which results in $\varepsilon=\frac{\left(1+1 / \delta_{c}\right)}{\hat{\kappa}_{c} \psi_{c}} \sqrt{\frac{n(2+\theta) \lambda_{\max }(Z)}{\theta}}(\|B\| \mu+\eta)$. Other terms are the same as before.

\section{Case study}

In this section we provide two case studies to illustrate our results and show their effectiveness in comparison with the existing compositional results by Swikir et al. (2018). We first apply our results to the temperature regulation in a circular building by constructing compositionally a finite abstraction of a network containing $n \geq 3$ rooms, each equipped with a heater. Then we apply the proposed techniques to a fully connected network to show its applicability to strongly connected networks as well. The construction of symbolic models and controllers are performed using tool SCOTS (Rungger \& Zamani, 2016) on a PC with Intel i7@3.4 GHz CPU and 16 GB of RAM.

\subsection{Room temperature control}

The evolution of the temperature $\mathbf{T}$ of all rooms is described by the interconnected discrete-time model:

$\Sigma:\left\{\begin{aligned} \mathbf{T}(k+1) & =A \mathbf{T}(k)+\beta T_{E}+\pi T_{h} v(k), \\ \mathbf{y}(k) & =\mathbf{T}(k),\end{aligned}\right.$

adapted from Meyer et al. (2018), where $A \in \mathbb{R}^{n \times n}$ is a matrix with elements $\{A\}_{i i}=\left(1-2 \alpha-\beta-\pi v_{i}(k)\right),\{A\}_{i(i+1)}=$ $\{A\}_{(i+1) i}=\{A\}_{1 n}=\{A\}_{n 1}=\alpha, \forall i \in[1 ; n-1]$, and all other elements are identically zero, $\mathbf{T}(k)=\left[\mathbf{T}_{1}(k) ; \ldots ; \mathbf{T}_{n}(k)\right], v(k)=$ $\left[v_{1}(k) ; \ldots ; v_{n}(k)\right], T_{E}=\left[T_{e 1} ; \ldots ; T_{e n}\right]$, where $v_{i}(k), \forall i \in[1 ; n]$,

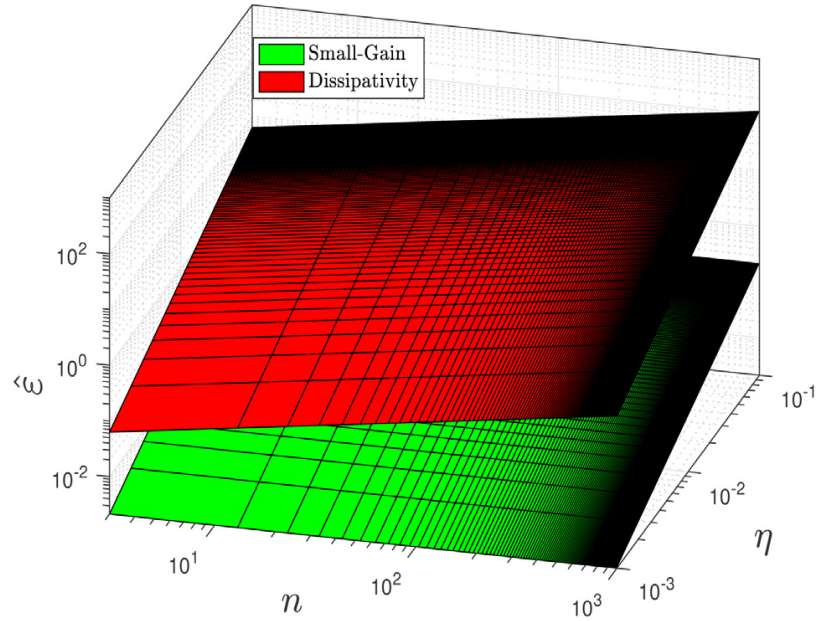

Fig. 2. Temperature control: Comparison of errors in (2.7) resulted from our approach based on small-gain condition with those resulted from the approach proposed by Swikir et al. (2018) based on dissipativity-type condition for different values of $n \geq 3$ and $\eta_{i}$.

are taking values in $[0,0.6]$. The other parameters are as follows: $\forall i \in[1 ; n], T_{e i}=-1{ }^{\circ} \mathrm{C}$ is the outside temperature, $T_{h}=50^{\circ} \mathrm{C}$ is the heater temperature, and the conduction factors are given by $\alpha=0.45, \beta=0.045$, and $\pi=0.09$.

Now, by introducing $\Sigma_{i}$ described by $\Sigma_{i}:\left\{\begin{aligned} \mathbf{T}_{i}(k+1) & =a \mathbf{T}_{i}(k)+d \omega_{i}(k)+\beta T_{e i}+\pi T_{h} v_{i}(k), \\ \mathbf{y}_{i}(k) & =\mathbf{T}_{i}(k),\end{aligned}\right.$

one can readily verify that $\Sigma=\mathcal{I}_{0_{n}}\left(\Sigma_{1}, \ldots, \Sigma_{n}\right)$, where $a=$ $1-2 \alpha-\beta-\pi v_{i}(k), d=[\alpha ; \alpha]^{T}$, and $\omega_{i}(k)=\left[\mathbf{y}_{i-1}(k) ; \mathbf{y}_{i+1}(k)\right]$ (with $\mathbf{y}_{0}=\mathbf{y}_{n}$ and $\left.\mathbf{y}_{n+1}=\mathbf{y}_{1}\right)$. Note that for any $i \in[1 ; n]$, conditions (4.1) and (4.2) are satisfied with $\mathcal{G}_{i}\left(T_{i}, \hat{T}_{i}\right)=\left\|T_{i}-\hat{T}_{i}\right\|, \mathcal{H}_{i} \equiv 0$, $\underline{\alpha}_{i}(s)=\bar{\alpha}_{i}(s)=s, \kappa_{i}(s)=(1-a) s, \gamma_{\text {iint }}(s)=\alpha s$, and $\gamma_{\text {iext }} \equiv$ 0 . Furthermore, (4.4) is satisfied with $\hat{\gamma}=\mathcal{I}_{d}$. Consequently, $\mathcal{G}_{i}\left(T_{i}, \hat{T}_{i}\right)=\left\|T_{i}-\hat{T}_{i}\right\|$ is an alternating simulation function from $\hat{\Sigma}_{i}$, constructed as in Definition 4.2 , to $\Sigma_{i}$.

Let, $\forall i \in[1 ; n]$, the $\mathcal{K}_{\infty}$ functions $\lambda_{i}, \psi_{i}$, and $\hat{\kappa}_{i}$ in the proof of Theorem 4.3 be as follows: $\lambda_{i}=\mathcal{I}_{d}, \psi_{i}(s)=0.99 \mathrm{~s}, \hat{\kappa}_{i}=$ $\kappa_{i}$. Since we have $\gamma_{i j}(s)<\mathcal{I}_{d}, \forall i, j \in[1 ; n], i \neq j$ and for any $n \geq 3$, the small-gain condition (3.7) is satisfied without any restriction on the number of rooms. Using the results in Theorem 3.4 with $\delta_{i}^{-1}=\mathcal{I}_{d}, \forall i \in[1 ; n]$, one can verify that $V(T, \hat{T})=\max _{i}\left\{\left\|T_{i}-\hat{T}_{i}\right\|\right\}$ is an alternating simulation function from $\Sigma=\mathcal{I}_{0_{n}}\left(\Sigma_{1}, \ldots, \hat{\Sigma}_{n}\right)$ to $\Sigma$ satisfying conditions (2.5) and (2.6) with $\tilde{\sigma}(s)=\max \left\{\left(1-(1-a) 10^{-2}\right) s, \frac{2.02 \alpha}{1-a} s\right\}, \tilde{\alpha}(s)=s$, $\tilde{\rho}_{\text {ext }}(s)=0 \forall s \in \mathbb{R}_{\geq 0}, \tilde{\varepsilon}=\max _{i}\left\{\frac{2.02 \eta_{i}}{1-a}\right\}, \forall i \in[1 ; N]$, where $\eta_{i}$ is the state set quantization parameter of abstraction $\hat{\Sigma}_{i}$.

Remark that, to have a fair comparison with the compositional technique proposed by Swikir et al. (2018), we have assumed that $\hat{\mathbb{Y}}_{j i}=\hat{\mathbb{W}}_{i j}$, i.e. $\hat{\varpi}_{i j}=0, \forall i, j \in[1 ; n], i \neq j$. For the fair comparison, we compute error $\hat{\varepsilon}$ in the $\hat{\varepsilon}$-approximate alternating simulation relation as in (2.7) based on the dissipativity approach introduced by Swikir et al. (2018) and the small-gain approach introduced here. This error represents the mismatch between the output behavior of the concrete interconnected system $\Sigma$ and that of its finite abstraction $\hat{\Sigma}$. We evaluate $\hat{\varepsilon}$ for different number of subsystems $n$ and different values of the state set quantization parameters $\eta_{i}$ for abstractions $\hat{\Sigma}_{i} \forall i \in[1 ; n]$ as in Fig. 2. As shown, the small-gain approach results in less mismatch errors than those obtained using the dissipativity based approach proposed by Swikir et al. (2018). The reason is that the error in (2.7) is 


$$
\begin{aligned}
& V\left(x_{d}, \hat{x}_{d}\right)=\left(\left(A x+B u+D w-(A \hat{x}+B \hat{u}+D \hat{w})+(A \hat{x}+B \hat{u}+D \hat{w})-\hat{x}_{d}\right)^{T} Z\right. \\
& \left(A x+B u+D w-(A \hat{x}+B \hat{u}+D \hat{w})+(A \hat{x}+B \hat{u}+D \hat{w})-\hat{x}_{d}\right)^{\frac{1}{2}} \\
& =\left((x-\hat{x})^{T}(A+B K)^{T} Z(A+B K)(x-\hat{x})+(w-\hat{w})^{T} D^{T} Z D(w-\hat{w})+2(w-\hat{w})^{T} D^{T} Z F\right. \\
& \left.+2(x-\hat{x})^{T}(A+B K)^{T} Z D(w-\hat{w})+2(x-\hat{x})^{T}(A+B K)^{T} Z F+F^{T} Z F\right)^{\frac{1}{2}} \\
& \leq\left((x-\hat{x})^{T}(A+B K)^{T} Z(A+B K)(x-\hat{x})+(w-\hat{w})^{T} D^{T} Z D(w-\hat{w})+2\left\|(w-\hat{w})^{T} D^{T} \sqrt{Z}\right\|_{2}\|\sqrt{Z} F\|_{2}\right. \\
& +2\left\|(x-\hat{x})^{T}(A+B K)^{T} \sqrt{Z}\right\|_{2}\|\sqrt{Z} F\|_{2}+2\left\|(x-\hat{x})^{T}(A+B K)^{T} \sqrt{Z}\right\|_{2}\|\sqrt{Z} D(w-\hat{w})\|_{2} \\
& \left.+n \lambda_{\max }(Z) \eta^{2}\right)^{\frac{1}{2}} \\
& \leq\left((x-\hat{x})^{T}(A+B K)^{T} Z(A+B K)(x-\hat{x})+2 \theta\left\|(x-\hat{x})^{T}(A+B K)^{T} \sqrt{Z}\right\|_{2}^{2}+(w-\hat{w})^{T} D^{T} Z D(w-\hat{w})\right. \\
& \left.+\frac{\left\|(w-\hat{w})^{T} D^{T} \sqrt{Z}\right\|_{2}^{2}}{\theta}+2 \frac{\|\sqrt{Z} F\|_{2}^{2}}{\theta}+\theta\left\|(w-\hat{w})^{T} D^{T} \sqrt{Z}\right\|_{2}^{2}+n \lambda_{\max }(Z) \eta^{2}\right)^{\frac{1}{2}} \\
& \leq\left((1+2 \theta)(x-\hat{x})^{T}(A+B K)^{T} Z(A+B K)(x-\hat{x})+\frac{\left(1+\theta+\theta^{2}\right)(w-\hat{w})^{T} D^{T} Z D(w-\hat{w})}{\theta}\right. \\
& \left.+\frac{n(2+\theta) \lambda_{\max }(Z) \eta^{2}}{\theta}\right)^{\frac{1}{2}} \\
& \leq \sqrt{\kappa_{c}} V(x, \hat{x})+\sqrt{\frac{1+\theta+\theta^{2}}{\theta}}\|\sqrt{Z} D\|_{2}\|w-\hat{w}\|_{2}+\sqrt{\frac{n(2+\theta) \lambda_{\max }(Z)}{\theta}} \eta \\
& \leq \sqrt{\kappa_{c}} V(x, \hat{x})+\sqrt{p \frac{1+\theta+\theta^{2}}{\theta}}\|\sqrt{Z} D\|_{2}\|w-\hat{w}\|+\sqrt{\frac{n(2+\theta) \lambda_{\max }(Z)}{\theta}} \eta \\
& \leq\left(1-\hat{\kappa}_{c}\right) V(x, \hat{x})+\sqrt{p \frac{1+\theta+\theta^{2}}{\theta}}\|\sqrt{Z} D\|_{2}\|w-\hat{w}\|+\sqrt{\frac{n(2+\theta) \lambda_{\max }(Z)}{\theta}} \eta . \\
& V\left(x_{d}, \hat{x}_{d}\right) \leq \max \left\{\tilde{\kappa}\left((x-\hat{x})^{T} Z(x-\hat{x})\right)^{\frac{1}{2}}, \frac{\left(1+\delta_{c}\right)}{\hat{\kappa}_{c} \psi_{c}} \sqrt{p \frac{\left(1+\theta+\theta^{2}\right)}{\theta}}\|\sqrt{Z} D\|_{2}\|w-\hat{w}\|, \frac{\left(1+1 / \delta_{c}\right)}{\hat{\kappa}_{c} \psi_{c}} \sqrt{\frac{n(2+\theta) \lambda_{\max }(Z)}{\theta}} \eta\right\}
\end{aligned}
$$

\section{Box II.}

computed based on the maximum of the errors between concrete subsystems and their finite abstractions instead of being a linear combination of them which is the case in Swikir et al. (2018). Hence, by increasing the number of subsystems, our error does not change here whereas the error computed by the dissipativity based approach proposed by Swikir et al. (2018) will increase as shown in Fig. 2.

Now, we synthesize a controller for $\Sigma$ via abstractions $\hat{\Sigma}_{i}$ such that the temperature of each room is maintained in the comfort zone $\mathcal{S}=[19,21]$. The idea here is to design local controllers for abstractions $\hat{\Sigma}_{i}$, and then refine them to concrete subsystems $\Sigma_{i}$. To do so, the local controllers are synthesized while assuming that the other subsystems meet their safety specifications. This approach, called assume-guarantee reasoning, allows for the compositional synthesis of controllers as well. The computation times for constructing abstractions and synthesizing controllers for $\Sigma_{i}$ are $0.048 \mathrm{~s}$ and $0.001 \mathrm{~s}$, respectively. Fig. 3 shows the state trajectories of the closed-loop system $\Sigma$, consisting of 1000 rooms, under control inputs $u_{i}$ with the state and input quantization parameters $\eta_{i}=0.01$ and $\mu_{i}=0.01, \forall i \in[1 ; 1000]$, respectively.

\subsection{Fully connected network}

In order to show the applicability of our approach to strongly connected networks, we consider a nonlinear control system $\Sigma$

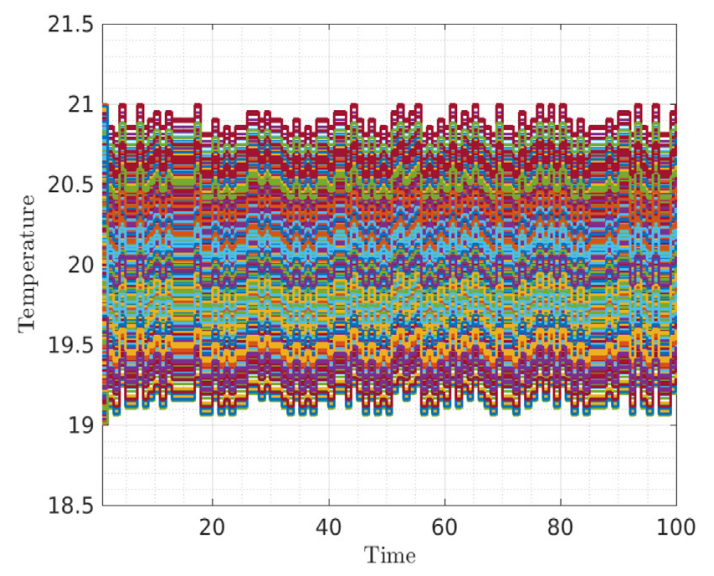

Fig. 3. State trajectories of the closed-loop system $\Sigma$ consisting of 1000 rooms.

described by

$\Sigma:\left\{\begin{aligned} \mathbf{x}(k+1) & =A \mathbf{x}(k)+\varphi(x)+v(k), \\ \mathbf{y}(k) & =\mathbf{x}(k),\end{aligned}\right.$

where $A=I_{n}-\tau L$ for some Laplacian matrix $L \in \mathbb{R}^{n \times n}$ of an undirected graph (Godsil \& Royle, 2001), and constant $0<$ $\tau<1 / \Delta$, where $\Delta$ is the maximum degree of the graph (Godsil 
\& Royle, 2001). Moreover $\mathbf{x}(k)=\left[\mathbf{x}_{1}(k) ; \ldots ; \mathbf{x}_{n}(k)\right], v(k)=$ $\left[v_{1}(k) ; \ldots ; v_{n}(k)\right]$, and $\varphi(x)=\left[\varphi_{1}\left(x_{1}\right) ; \ldots ; \varphi_{n}\left(x_{n}\right)\right]$, where $\varphi_{i}\left(x_{i}\right)=$ $\sin \left(x_{i}\right), \forall i \in[1 ; n]$. Assume $L$ is the Laplacian matrix of a complete graph:

$L=\left[\begin{array}{ccccc}n-1 & -1 & \ldots & \ldots & -1 \\ -1 & n-1 & -1 & \ldots & -1 \\ -1 & -1 & n-1 & \ldots & -1 \\ \vdots & & \ddots & \ddots & \vdots \\ -1 & \ldots & \ldots & -1 & n-1\end{array}\right]$.

Now, by introducing $\Sigma_{i}$ described by

$\Sigma_{i}:\left\{\begin{aligned} \mathbf{x}_{i}(k+1) & =a_{i} \mathbf{x}_{i}(k)+\varphi_{i}\left(x_{i}\right)+d_{i} \omega_{i}(k)+v_{i}(k), \\ \mathbf{y}_{i}(k) & =\mathbf{x}_{i}(k),\end{aligned}\right.$

where $a_{i}=\{A\}_{i i}, \omega_{i}(k)=\left[\mathbf{y}_{i 1} ; \ldots ; \mathbf{y}_{i(i-1)} ; \mathbf{y}_{i(i+1)} ; \ldots ; \mathbf{y}_{i n}\right], d_{i}=$ $\left[\{A\}_{i 1} ; \ldots ;\{A\}_{i(i-1)} ;\{A\}_{i(i+1)} ; \ldots ;\{A\}_{i n}\right]^{T}$, one can readily verify that $\Sigma=\mathcal{I}_{0_{n}}\left(\Sigma_{1}, \ldots, \Sigma_{n}\right)$. Clearly, for any $i \in[1 ; n]$, conditions (4.1) and (4.2) are satisfied with $\mathcal{G}_{i}\left(x_{i}, \hat{x}_{i}\right)=\left\|x_{i}-\hat{x}_{i}\right\|, \mathcal{H}_{i}\left(x_{i}\right)=$ $-c_{i} x_{i}$, where $\frac{a_{i}+1}{2}<c_{i}<a_{i}+1, \underline{\alpha}_{i}(s)=\bar{\alpha}_{i}(s)=s, \kappa_{i}(s)=$ $\left(1-\left(1+a_{i}-c_{i}\right)\right) s, \gamma_{\text {iint }}(s)=\left\|d_{i}\right\| s$, and $\gamma_{\text {iext }}(s)=0, \forall s \in \mathbb{R}_{\geq 0}$. Note that (4.4) is satisfied with $\hat{\gamma}=\mathcal{I}_{d}$. Consequently, $\mathcal{G}_{i}\left(x_{i}, \hat{x}_{i}\right)=$ $\left\|x_{i}-\hat{x}_{i}\right\|$ is an alternating simulation function from $\hat{\Sigma}_{i}$, constructed as in Definition 4.2, to $\Sigma_{i}$.

Fix $\tau=\frac{0.1}{\Delta}=\frac{0.1}{n-1}$, and let, $\forall i \in[1 ; n]$, the $\mathcal{K}_{\infty}$ functions $\lambda_{i}$, $\psi_{i}$, and $\hat{\kappa}_{i}$ in the proof of Theorem 4.3 be as follows: $\lambda_{i}=\mathcal{I}_{d}$, $\psi_{i}(s)=0.99 s, \hat{\kappa}_{i}=\kappa_{i}$. Since we have $\gamma_{i j}(s)<\mathcal{I}_{d}, \forall i, j \in$ $[1 ; n], i \neq j$, the small-gain condition (3.7) is satisfied without any restriction on the number of subsystems. Using the results in Theorem 3.4 with $\delta_{i}^{-1}=\mathcal{I}_{d}, \forall i \in[1 ; n]$, one can verify that $V(x, \hat{x})=\max _{i}\left\{\left\|x_{i}-\hat{x}_{i}\right\|\right\}$ is an alternating simulation function from $\hat{\Sigma}=\mathcal{I}_{0_{n}}\left(\hat{\Sigma}_{1}, \ldots, \hat{\Sigma}_{n}\right)$ to $\Sigma$ satisfying conditions (2.5) and (2.6) with $\tilde{\alpha}(s)=s, \tilde{\rho}_{\text {ext }}(s)=0, \forall s \in \mathbb{R}_{\geq 0}, \tilde{\varepsilon}=\max _{i}\left\{\frac{2.02 \eta_{i}}{1-\left(1+a_{i}-c_{i}\right)}\right\}$, $\tilde{\sigma}(s)=\max \left\{\max _{i}\left\{\left(1-\frac{\left(1-\left(1+a_{i}-c_{i}\right)\right)}{10^{2}}\right) s\right\}, \max _{i}\left\{\frac{2.02\left\|d_{i}\right\|}{1-\left(1+a_{i}-c_{i}\right)} s\right\}\right\}$, where $\eta_{i}$ is the state set quantization parameter of abstraction $\hat{\Sigma}_{i}$.

Similar to the previous case study, in order to compare our compositional technique to the one proposed by Swikir et al. $(2018)$, we have assumed that $\hat{\mathbb{Y}}_{j i}=\widehat{\mathbb{W}}_{i j}$, i.e. $\hat{\varpi}_{i j}=0, \forall i, j \in$ $[1 ; n], i \neq j$. A comparison of the error $\hat{\varepsilon}$ in (2.7) resulted from the dissipativity approach proposed by Swikir et al. (2018) and the small-gain approach introduced here is shown in Fig. 4. We compute $\hat{\varepsilon}$ for different number of subsystems $n$ and different values of the state set quantization parameters $\eta_{i}$ for abstractions $\hat{\Sigma}_{i}, \forall i \in[1 ; n]$. Clearly, the small-gain approach results in less mismatch errors than those obtained using the dissipativity based approach introduced by Swikir et al. (2018). The computation time for constructing abstractions for $\Sigma_{i}$ is $0.9 \mathrm{~s}$ after fixing $n=1000, \eta_{i}=0.01, \mu_{i}=0.01, x_{i} \in[0,10], v_{i} \in[0,1]$, $\forall i \in[1 ; n]$.

\section{Conclusion}

In this paper, we proposed a compositional framework for the construction of finite abstractions of interconnected discrete-time control systems. First, we used a notion of so-called alternating simulation functions in order to construct compositionally an overall alternating simulation function that is used to quantify the error between the output behavior of the overall interconnected concrete system and the one of its finite abstraction. Furthermore, we provided a technique to construct finite abstractions together with their corresponding alternating simulation functions for discrete-time control systems under incremental input-to-state stabilizability property. Finally, we illustrated the

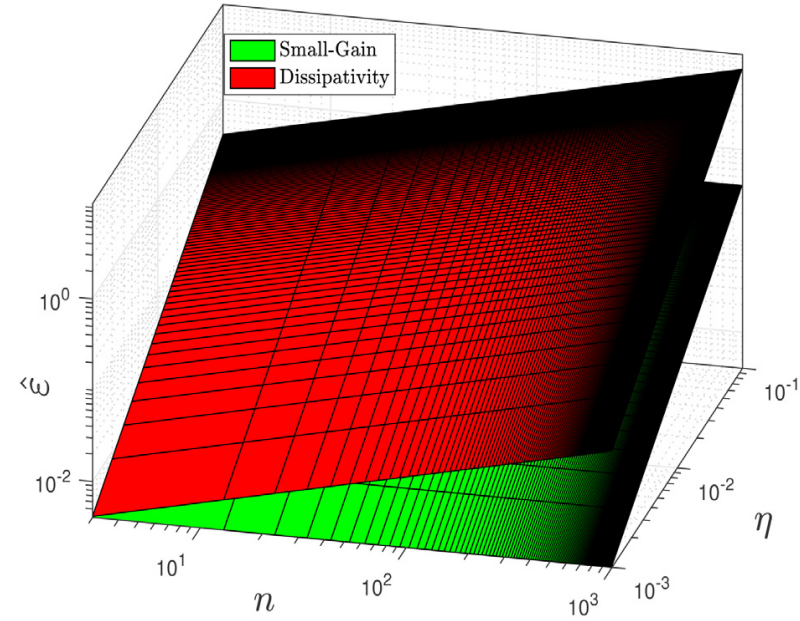

Fig. 4. Fully connected network: Comparison of errors in (2.7) resulted from our approach based on small-gain condition with those resulted from the approach proposed by Swikir et al. (2018) based on dissipativity-type condition for different values of $n \geq 1$ and $\eta_{i}$.

proposed results by constructing finite abstractions of two networks of (linear and nonlinear) discrete-time control systems and their corresponding alternating simulation functions in a compositional fashion. We elucidated the effectiveness of our compositionality results in comparison with the existing ones using dissipativity-type reasoning.

\section{References}

Angeli, D. (2002). A Lyapunov approach to incremental stability properties. IEEE Transactions on Automatic Control, 47(3), 410-421.

Antsaklis, P. J., \& Michel, A. N. (2007). A linear systems primer. Birkhäuser.

Arcak, M., Meissen, C., \& Packard, A. (2016). Springerbriefs in electrical and computer engineering, Networks of dissipative systems. Springer International Publishing.

Dashkovskiy, S., Rüffer, B., \& Wirth, F. (2010). Small gain theorems for large scale systems and construction of ISS Lyapunov functions. SIAM Journal on Control and Optimization, 48(6), 4089-4118.

Eaves, B. C. (1972). Homotopies for computation of fixed points. Mathematical Programming, 3(1), 1-22.

Girard, A., \& Pappas, G. J. (2009). Hierarchical control system design using approximate simulation. Automatica, 45(2), 566-571.

Godsil, C., \& Royle, G. (2001). Graduate texts in mathematics: vol. 207, Algebraic graph theory. Springer.

Henzinger, T. A., Shaz, Q., \& Rajamani, S. K. (1998). You assume, we guarantee: Methodology and case studies. In Proceedings of international conference on computer aided verification (pp. 440-451).

Hussein, O., Ames, A., \& Tabuada, P. (2017). Abstracting partially feedback linearizable systems compositionally. IEEE Control Systems Letters, 1(2), 227-232.

Jiang, Z.-P., Mareels, I. M., \& Wang, Y. (1996). A Lyapunov formulation of the nonlinear small-gain theorem for interconnected ISS systems. Automatica 32(1), 1211-1215.

Kellett, C. (2014). A compendium of comparison function results. Mathematics of Control, Signals, and Systems, 26(3), 339-374.

Kim, E. S., Arcak, M., \& Zamani, M. (2018). Constructing control system abstractions from modular components. In Proceedings of the 21st international conference on hybrid systems: Computation and control (pp. 137-146). ACM.

Maler, O., Pnueli, A., \& Sifakis, J. (1995). On the synthesis of discrete controllers for timed systems. In Proceedings of the 12th symposium on theoretical aspects of computer science (pp. 229-242).

Mallik, K., Schmuck, A., Soudjani, S., \& Majumdar, R. (2019). Compositional synthesis of finite state abstractions. IEEE Transactions on Automatic Control, 64(6), 2629-2636.

Meyer, P. J., Girard, A., \& Witrant, E. (2018). Compositional abstraction and safety synthesis using overlapping symbolic models. IEEE Transactions on Automatic Control, 63(6), 1835-1841. 
Noroozi, N., Geiselhart, R., Grüne, L., Rüffer, B. S., \& Wirth, F. R. (2018). Nonconservative discrete-time ISS small-gain conditions for closed sets. IEEE Transactions on Automatic Control, 63(5), 1231-1242.

Pola, G., Pepe, P., \& Benedetto, M. D. D. (2016). Symbolic models for networks of control systems. IEEE Transactions on Automatic Control, 61(11), 3663-3668.

Pola, G., \& Tabuada, P. (2009). Symbolic models for nonlinear control systems: Alternating approximate bisimulations. SIAM Journal on Control and Optimization, 48(2), 719-733.

Rockafellar, R. T., \& Wets, R. (2009). Variational analysis, Vol. 317. Springer-Verlag.

Ruffer, B. S. (2007). Monotone dynamical systems, graphs, and stability of largescale interconnected systems. In Fachbereich 3, Mathematik und informatik (Ph.D. thesis), Germany: Universität Bremen.

Rungger, M., \& Zamani, M. (2016). SCOTS: A tool for the synthesis of symbolic sontrollers. In Proceedings of the 19th international conference on hybrid systems: computation and control (pp. 99-104).

Rungger, M., \& Zamani, M. (2018). Compositional construction of approximate abstractions of interconnected control systems. IEEE Transactions on Control of Network Systems, 5(1), 116-127.

Swikir, A., Girard, A., \& Zamani, M. (2018). From dissipativity theory to compositional synthesis of symbolic models. In Proceedings of the 4th Indian control conference (pp. 30-35)

Tabuada, P. (2009). Verification and control of hybrid systems: A symbolic approach (1st ed.). Springer Publishing Company, Incorporated.

Tazaki, Y., \& Imura, J. I. (2008). Bisimilar finite abstractions of interconnected systems. In Proceedings of the 11th international conference on hybrid systems: computation and control (pp. 514-527). Springer Berlin Heidelberg.

Thomas, W. (1995). On the synthesis of strategies in infinite games. In LNCS: vol. 900, Proceedings of the 12th annual symposium on theoretical aspects of computer science (pp. 1-13). Springer Berlin Heidelberg.

Tran, D. N., Rüffer, B. S., \& Kellett, C. M. (2016). Incremental stability properties for discrete-time systems. In Proceedings of the 55th conference on decision and control (pp. 477-482)

Zamani, M., Mohajerin Esfahani, P., Majumdar, R., Abate, A., \& Lygeros, J. (2014). Symbolic control of stochastic systems via approximately bisimilar finite abstractions. IEEE Transactions on Automatic Control, 59(12) 3135-3150.

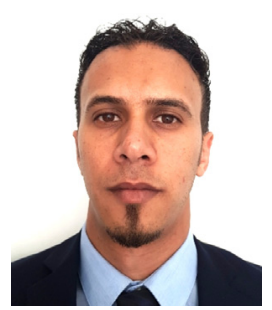

Abdalla Swikir is currently a $\mathrm{PhD}$ candidate in the Department of Electrical and Computer Engineering at Technical University of Munich (TUM), Germany, since November 2016. He is also a faculty member in the Electrical Engineering Department, Omar Al-Mukhtar University, Albyada, Libya. He received a B.Sc. degree in Electrical Engineering in August 2008 from Omar Al-Mukhtar University, Albyada, Libya, and an M.Sc. degree in Electrical Engineering in December 2015 from Ohio State University, Columbus, OH, USA. From January 2010 to April 2013 he was a teaching assistant in the Department of Electrical Engineering at Omar Al-Mukhtar University, Albyada, Libya.

His research interests are formal synthesis, Symbolic models, and compositional methods of Large-Scale Cyber-Physical Systems.

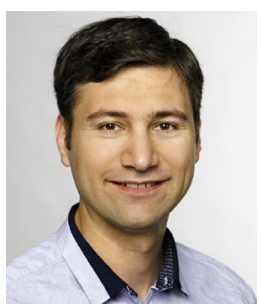

Majid Zamani is an Assistant Professor in the Computer Science Department at the University of Colorado Boulder, USA. He is also a guest professor in the Computer Science Department at the Ludwig Maximilian University of Munich. He received a B.Sc. degree in Electrical Engineering in 2005 from Isfahan University of Technology, Iran, an M.Sc. degree in Electrical Engineering in 2007 from Sharif University of Technology, Iran, an MA degree in Mathematics and a Ph.D. degree in Electrical Engineering both in 2012 from University of California, Los Angeles, USA. Between September 2012 and December 2013 he was a postdoctoral researcher at the Delft Centre for Systems and Control, Delft University of Technology, Netherlands. From May 2014 to January 2019 he was an Assistant Professor in the Department of Electrical and Computer Engineering at the Technical University of Munich, Germany. From December 2013 to April 2014 he was an Assistant Professor in the Design Engineering Department, Delft University of Technology, Netherlands. He received an ERC starting grant award from the European Research Council in 2018.

His research interests include verification and control of hybrid systems, embedded control software synthesis, networked control systems, and incremental properties of nonlinear control systems. 